\title{
KNASTER AND FRIENDS I: CLOSED COLORINGS AND PRECALIBERS
}

\author{
CHRIS LAMBIE-HANSON AND ASSAF RINOT
}

\begin{abstract}
The productivity of the $\kappa$-chain condition, where $\kappa$ is a regular, uncountable cardinal, has been the focus of a great deal of set-theoretic research. In the $1970 \mathrm{~s}$, consistent examples of $\kappa$-cc posets whose squares are not $\kappa$-cc were constructed by Laver, Galvin, Roitman and Fleissner. Later, ZFC examples were constructed by Todorcevic, Shelah, and others. The most difficult case, that in which $\kappa=\aleph_{2}$, was resolved by Shelah in 1997.

In this work, we obtain analogous results regarding the infinite productivity of strong chain conditions, such as the Knaster property. Among other results, for any successor cardinal $\kappa$, we produce a ZFC example of a poset with precaliber $\kappa$ whose $\omega^{\text {th }}$ power is not $\kappa$-cc. To do so, we carry out a systematic study of colorings satisfying a strong unboundedness condition. We prove a number of results indicating circumstances under which such colorings exist, in particular focusing on cases in which these colorings are moreover closed.
\end{abstract}

\section{INTRODUCTION}

Questions about the productivity of the $\kappa$-chain condition for regular, uncountable cardinals $\kappa$ have led to a great deal of set-theoretic research. (For an overview, see [Rin14].) A central tool that arose in these investigations, implicit in work of Galvin [Gal80] and isolated by Shelah [She88], is the following principle asserting the existence of rather complicated colorings. (For unfamiliar notation, in particular our conventions regarding the expression $[\mathcal{A}]^{2}$, see the Notation subsection at the end of the Introduction.)

Definition 1.1 (Shelah, [She88]). $\operatorname{Pr}_{1}(\kappa, \kappa, \theta, \chi)$ asserts the existence of a coloring $c:[\kappa]^{2} \rightarrow \theta$ such that for every $\chi^{\prime}<\chi$, every family $\mathcal{A} \subseteq[\kappa] \chi^{\prime}$ consisting of $\kappa$-many pairwise disjoint sets, and every $i<\theta$, there is $(a, b) \in[\mathcal{A}]^{2}$ such that $c[a \times b]=\{i\}$.

The primary connection between this principle and the productivity of the $\kappa$ chain condition stems from the fact that, if $\kappa$ is a regular cardinal and $\operatorname{Pr}_{1}(\kappa, \kappa, 2, \omega)$ holds, then the $\kappa$-chain condition fails to be productive.

The work in this paper is motivated in large part by questions concerning the infinite productivity of the $\kappa$-chain condition and its strengthenings, in particular the $\kappa$-Knaster condition. We introduce and study the following principle, which plays a role in questions about the infinite productivity of the $\kappa$-Knaster condition that is analogous to the role played by $\operatorname{Pr}_{1}(\kappa, \kappa, \theta, \chi)$ in questions about the productivity of the $\kappa$-chain condition.

Date: December 5, 2018.

2010 Mathematics Subject Classification. Primary 03E35; Secondary 03E05, 03E75, 06E10.

Key words and phrases. Knaster, precaliber, closed coloring, unbounded function, stationary reflection, square.

This research was partially supported by the Israel Science Foundation (grant \#1630/14). 
Definition 1.2. $\mathrm{U}(\kappa, \mu, \theta, \chi)$ asserts the existence of a coloring $c:[\kappa]^{2} \rightarrow \theta$ such that for every $\chi^{\prime}<\chi$, every family $\mathcal{A} \subseteq[\kappa]^{\chi^{\prime}}$ consisting of $\kappa$-many pairwise disjoint sets, and every $i<\theta$, there exists $\mathcal{B} \in[\mathcal{A}]^{\mu}$ such that $\min (c[a \times b])>i$ for all $(a, b) \in[\mathcal{B}]^{2}$.

Remark 1. Note two conceptual differences between Definitions 1.1 and 1.2:

(1) The second coordinate in the principle $\mathrm{U}(\kappa, \mu, \theta, \chi)$ plays a different role from the second coordinate in the principle $\operatorname{Pr}_{1}(\kappa, \lambda, \theta, \chi)$. This is the reason we choose to only define the case $\lambda=\kappa$.

(2) While $\operatorname{Pr}_{1}(\kappa, \kappa, \theta, \chi)$ implies $\operatorname{Pr}_{1}\left(\kappa, \kappa, \theta^{\prime}, \chi\right)$ for $\theta^{\prime}<\theta$, the principle $\mathrm{U}(\ldots)$ offers no monotonicity in the third coordinate. Indeed, the instance $\mathrm{U}(\kappa, \kappa, \kappa, \kappa)$ is a trivial consequence of ZF.

It is worth pointing out that certain instances of the above principle are implicit in previous works. We mention a few examples here.

- Implicit in the conclusion of [She94, Claim 4.9] is the instance $\mathrm{U}(\kappa, 2, \omega, \chi)$.

- Implicit in the proof of [She97, Claim 4.1] is a proof of the fact that for every infinite regular cardinal $\lambda, \mathrm{U}\left(\lambda^{+}, 2, \lambda, \lambda\right)$ holds.

- Implicit in [Tod07, Theorem 6.3.6] is the statement that, for every infinite cardinal $\lambda, \mathrm{U}\left(\lambda^{+}, \lambda^{+}, \omega, \operatorname{cf}(\lambda)\right)$ holds.

- Implicit in the proof of [FR17, Lemma 3.4] is the fact that any witness to $\operatorname{Pr}_{1}(\kappa, \kappa, \theta, \chi)$ is also a witness to $\mathrm{U}(\kappa, 2, \theta, \chi)$.

1.1. Summary of results. The results in this paper primarily fall into two classes. The first consists of results asserting that, under appropriate circumstances, certain instances of $\mathrm{U}(\ldots)$ provably hold. The second consists of applications of $\mathrm{U}(\ldots)$ to questions regarding the infinite productivity of strengthenings of the $\kappa$-chain condition and generalizations of Martin's Axiom to higher cardinals. We preview some of the prominent results here, beginning with those from the first class.

Theorem A. Suppose that $\chi, \theta<\kappa$ are infinite cardinals. If either of the two following hypotheses holds, then $\mathrm{U}(\kappa, \kappa, \theta, \chi)$ holds:

(1) $\square(\kappa)$ holds; or

(2) there exists a non-reflecting stationary subset of $E_{\geq \chi}^{\kappa}$.

In particular, if $\theta \leq \lambda$ are infinite, regular cardinals, then $\mathrm{U}\left(\lambda^{+}, \lambda^{+}, \theta, \lambda\right)$ holds.

Proof. This follows from Corollaries 4.10 and 4.12 and the fact that, if $\lambda$ is a regular cardinal, then $E_{\lambda}^{\lambda^{+}}$is a non-reflecting stationary set.

The previous result indicates that all possible instances of $\mathrm{U}(. .$.$) hold at suc-$ cessors of regular cardinals and also provides cases in which nontrivial instances of $\mathrm{U}(\ldots)$ hold at successors of singular cardinals and inaccessible cardinals. The next results provide further information in this direction.

Theorem B. If $\lambda$ is a singular cardinal and $\theta \leq \lambda$ is an infinite cardinal, then any one of the following hypotheses implies that $\mathrm{U}\left(\lambda^{+}, \lambda^{+}, \theta, \operatorname{cf}(\lambda)\right)$ holds:

(1) $2^{\lambda}=\lambda^{+}$;

(2) $\operatorname{Refl}\left(<\operatorname{cf}(\lambda), \lambda^{+}\right)$fails;

(3) there is a closed witness to $\mathrm{U}\left(\lambda^{+}, 2, \theta, 2\right)$;

(4) $\operatorname{cf}(\theta)<\operatorname{cf}(\lambda)$ and $2^{\operatorname{cf}(\lambda)}<\lambda$;

(5) $\operatorname{cf}(\theta)=\operatorname{cf}(\lambda)$; 
(6) $\operatorname{cf}(\theta)=\omega$.

Proof. (1) follows from Theorem 4.15, (2)-(4) follow from Theorem 4.16, (5) follows from Corollary 4.13, and (6) follows from Corollary 4.8.

Theorem C. If $\kappa$ is an inaccessible cardinal, $\chi<\kappa$ is an infinite cardinal, and there is a stationary subset of $E_{\geq \chi}^{\kappa}$ that does not reflect at any inaccessible cardinal, then $\mathrm{U}(\kappa, \kappa, \theta, \chi)$ holds for every infinite cardinal $\theta<\kappa$.

Proof. This follows from Theorem 4.18.

Observe that the presence of large cardinals places limits on the extent to which $\mathrm{U}(\ldots)$ holds at inaccessible cardinals or successors of singular cardinals. In particular, it is immediate that, if $\kappa$ is weakly compact, then $\mathrm{U}(\kappa, 2, \theta, 2)$ fails for every $\theta<\kappa$. We also show that, if $\lambda$ is a singular limit of strongly compact cardinals, then $\mathrm{U}\left(\lambda^{+}, 2, \theta, \operatorname{cf}(\lambda)^{+}\right)$fails for all $\theta \in \operatorname{Reg}(\lambda) \backslash\{\operatorname{cf}(\lambda)\}$.

We next turn to applications of $\mathrm{U}(\ldots)$. Our primary result regarding the infinite productivity of strong chain conditions is as follows and answers a question raised at the end of the Introduction of [LHL19]

Theorem D. Suppose that $\theta, \chi<\kappa$ are infinite regular cardinals, $\kappa$ is $(<\chi)$ inaccessible, and $\mathrm{U}(\kappa, \kappa, \theta, \chi)$ holds. Then there exists a $\chi$-directed closed poset $\mathbb{P}$ such that $\mathbb{P}^{\tau}$ is $\kappa$-Knaster for all $\tau<\min (\{\chi, \theta\})$, but $\mathbb{P}^{\theta}$ is not $\kappa$-cc. In particular, for every infinite successor cardinal $\kappa$, there exists a $\kappa$-Knaster poset $\mathbb{P}$ whose $\omega^{\text {th }}$ power is not $\kappa$-cc.

Proof. This follows from Lemma 3.3 and Corollary 4.8.

We also present an unpublished result of Inamdar [Ina17] indicating a fundamental limitation to generalizations of Martin's Axiom to higher cardinals. Implicit in Inamdar's proof was a use of $\mathrm{U}\left(\lambda^{+}, \lambda^{+}, \omega, \lambda\right)$ for regular, uncountable $\lambda$ that helped motivate some of the work in this paper.

1.2. Structure of the paper and its sequels. In Section 2, we present some basic facts about $\mathrm{U}(. .$.$) to lay the framework for further analysis. In particular,$ we consider some elementary implications and non-implications that exist between various instances of $\mathrm{U}(\ldots)$, prove some limitations placed on $\mathrm{U}(\ldots)$ by the existence of large cardinals, and we discuss some properties of trees derived from witnesses to $\mathrm{U}(\ldots)$.

In Section 3, we present the primary applications of the paper. Subsection 3.1 contains our results regarding $\mathrm{U}(. .$.$) and the infinite productivity of strengthenings$ of the $\kappa$-chain condition. In Subsection 3.2, we present the aforementioned result of Inamdar concerning generalizations of Martin's Axiom to higher cardinals.

In Section 4, we prove our results regarding circumstances under which instances of $U(. .$.$) necessarily hold. In all cases, our proofs will in fact yield witnesses to$ $\mathrm{U}(. .$.$) with certain closure properties that make them better-behaved. In Subsec-$ tion 4.1, we present some basic facts about these closed colorings. In Subsection 4.2, we review the necessary background concerning walks on ordinals, which provide our main tool for constructing witnesses to U(...). Subsection 4.3 contains our first construction of such witnesses, in particular yielding the fact that all possible instances of $\mathrm{U}(\ldots)$ hold at successors of regular cardinals. Subsections 4.4 and 4.5 contain further constructions at successors of singular cardinals and inaccessible cardinals, respectively. 
As the title of the paper suggests, it is the first paper in a series. In Part II, we introduce a new cardinal invariant for regular uncountable cardinals, the $C$-sequence number, which is intimately connected to the fourth parameter of $\mathrm{U}(. .$.$) as well as$ to various square principles. Considerations of the $C$-sequence number will allow us to obtain additional results regarding the existence of closed witnesses to $\mathrm{U}(\ldots)$. In Part III, we study the existence of subadditive witnesses to $\mathrm{U}(\ldots)$ and discuss applications of such subadditive witnesses to the infinite productivity of further strengthenings of the $\kappa$-chain condition and to topological matters, such as the question as to the tightness of the square of the sequential fan. The techniques of parts II and III will also allow us to prove independence results separating certain instances of $\mathrm{U}(\ldots)$ at inaccessible cardinals and successors of singular cardinals. Where relevant, we will make reference to results in Parts II and III that will provide further context for the results in this paper, though no knowledge of these papers is necessary for any of the results contained here.

1.3. Notation and conventions. Throughout the paper, $\kappa$ denotes a regular uncountable cardinal, and $\chi, \theta$, and $\mu$ denote cardinals $\leq \kappa$. We say that $\kappa$ is $(<\chi)$-inaccessible iff, for all $\lambda<\kappa$ and $\nu<\chi, \lambda^{\nu}<\kappa$. Reg denotes the class of infinite regular cardinals, and $\operatorname{Reg}(\kappa)$ denotes $\operatorname{Reg} \cap \kappa$. $E_{\chi}^{\kappa}$ denotes the set $\{\alpha<\kappa \mid$ $\operatorname{cf}(\alpha)=\chi\}$, and $E_{\geq \chi}^{\kappa}, E_{>\chi}^{\kappa}, E_{\neq \chi}^{\kappa}$, etc. are defined analogously. For a subset $S \subseteq \kappa$, we let $\operatorname{Tr}(S):=\left\{\alpha \in E_{>\omega}^{\kappa} \mid S \cap \alpha\right.$ is stationary in $\left.\alpha\right\}$; We say that $S$ is nonreflecting (resp. non-reflecting at inaccessibles) iff $\operatorname{Tr}(S)$ is empty (resp. contains no inaccessible cardinals). The principle $\operatorname{Refl}(<\theta, S)$ asserts that for every family $\mathcal{S}$ consisting of less than $\theta$-many stationary subsets of $S$, the set $\bigcap_{S \in \mathcal{S}} \operatorname{Tr}(S)$ is nonempty.

For an ideal $\mathcal{I}$ on $\kappa$, we write $\mathcal{I}^{+}:=\mathcal{P}(\kappa) \backslash \mathcal{I}$, and $\mathcal{I}^{*}:=\{\kappa \backslash X \mid X \in \mathcal{I}\}$.

For the definitions of the principles $\square\left(\kappa, \sqsubseteq_{\sigma}\right), \square(\kappa)$ and $\square_{\lambda}^{*}$, see [BR19], Definition 1.16, and the discussion following it. For the definitions of the principles $\diamond(S)$, $\boldsymbol{A}^{-}(S)$ and $(S)$, see [Rin11], Definitions 1.1, 1.18 and 2.15, respectively.

For a set of ordinals $a$, we write $\operatorname{ssup}(a):=\sup \{\alpha+1 \mid \alpha \in a\}, \operatorname{acc}^{+}(a):=\{\alpha<$ $\operatorname{ssup}(a) \mid \sup (a \cap \alpha)=\alpha>0\}, \operatorname{acc}(a):=a \cap \operatorname{acc}^{+}(a), \operatorname{nacc}(a):=a \backslash \operatorname{acc}(a)$, and $\operatorname{cl}(a):=a \cup \operatorname{acc}^{+}(a)$. For sets of ordinals, $a$ and $b$, we write $a<b$ if, for all $\alpha \in a$ and all $\beta \in b$, we have $\alpha<\beta$. For a set of ordinals $a$ and an ordinal $\beta$, we write $a<\beta$ instead of $a<\{\beta\}$ and $\beta<a$ instead of $\{\beta\}<a$.

For any set $\mathcal{A}$, we write $[\mathcal{A}]^{\chi}:=\{\mathcal{B} \subseteq \mathcal{A}|| \mathcal{B} \mid=\chi\}$ and $[\mathcal{A}]^{<\chi}:=\{\mathcal{B} \subseteq \mathcal{A} \mid$ $|\mathcal{B}|<\chi\}$. In particular, $[\mathcal{A}]^{2}$ consists of all unordered pairs from $\mathcal{A}$. In some scenarios, we will also be interested in ordered pairs from $\mathcal{A}$. In particular, if $\mathcal{A}$ is either an ordinal or a collection of sets of ordinals, then we will abuse notation and write $(a, b) \in[\mathcal{A}]^{2}$ to mean $\{a, b\} \in[\mathcal{A}]^{2}$ and $a<b$.

\section{Preliminary Results}

In this section, we present some basic results regarding $\mathrm{U}(\kappa, \mu, \theta, \chi)$. We begin by cataloging some implications that exist between various incarnations of the coloring principles under consideration. The following proposition is immediate.

Proposition 2.1. (1) $\mathrm{U}(\kappa, \kappa, \kappa, \kappa)$ holds.

(2) If $\operatorname{cf}\left(\theta^{\prime}\right)=\operatorname{cf}(\theta)$, then $\mathrm{U}(\kappa, \mu, \theta, \chi)$ holds iff $\mathrm{U}\left(\kappa, \mu, \theta^{\prime}, \chi\right)$ holds.

(3) For all $\mu^{\prime} \leq \mu$ and $\chi^{\prime} \leq \chi, \mathrm{U}(\kappa, \mu, \theta, \chi)$ entails $\mathrm{U}\left(\kappa, \mu^{\prime}, \theta, \chi^{\prime}\right)$. 
(4) If $\chi$ is a limit cardinal and $c:[\kappa]^{2} \rightarrow \theta$ witnesses $\mathrm{U}\left(\kappa, \mu, \theta, \chi^{\prime}\right)$ for all $\chi^{\prime}<\chi$, then $c$ witnesses $\mathrm{U}(\kappa, \mu, \theta, \chi)$, as well.

(5) If $\mathrm{U}(\kappa, 2, \theta, \chi)$ and $\kappa \rightarrow(\kappa, \mu)^{2}$ both hold, then $\mathrm{U}(\kappa, \mu, \theta, \chi)$ holds, as well. In particular, $\mathrm{U}(\kappa, 2, \theta, \chi)$ entails $\mathrm{U}(\kappa, \omega, \theta, \chi)$.

(6) If $\chi<\kappa$, then $\mathrm{U}(\kappa, \mu, \theta, \chi)$ holds iff there exists a coloring $c:[\kappa]^{2} \rightarrow \theta$ such that, for every family $\mathcal{A} \subseteq[\kappa]^{<\chi}$ consisting of $\kappa$-many pairwise disjoint sets, and for every $i<\theta$, there exists $\mathcal{B} \in[\mathcal{A}]^{\mu}$ such that $\min (c[a \times b])>i$ for all $(a, b) \in[\mathcal{B}]^{2}$.

Remark 2. For Clause (5), recall that $\kappa \rightarrow(\kappa, \mu)^{2}$ stands for the assertion that for every coloring $c:[\kappa]^{2} \rightarrow 2$, either there exists $A \in[\kappa]^{\kappa}$ such that $c^{\prime \prime}[A]^{2}=\{0\}$, or there exists $B \in[\kappa]^{\mu}$ such that $c^{\text {" }}[B]^{2}=\{1\}$. By a classic theorem of Dushnik and Miller, $\kappa \rightarrow(\kappa, \omega)^{2}$ holds for every infinite cardinal $\kappa$.

Because of Clauses (1) and (2) of the preceding Proposition, we shall focus throughout on the case in which $\theta \in \operatorname{Reg}(\kappa)$. We next note that instances of $\operatorname{Pr}_{1}(\ldots)$ easily yield instances of $\mathrm{U}(\ldots)$.

Lemma 2.2. Suppose that $\operatorname{Pr}_{1}(\kappa, \kappa, \theta, \chi)$ holds. Then $\mathrm{U}\left(\kappa, 2, \theta^{\prime}, \chi\right)$ holds for all $\theta^{\prime} \leq \theta$

Proof. Let $c:[\kappa]^{2} \rightarrow \theta$ witness $\operatorname{Pr}_{1}(\kappa, \kappa, \theta, \chi)$, and fix $\theta^{\prime} \leq \theta$. Define $c^{\prime}:[\kappa]^{2} \rightarrow \theta^{\prime}$ by setting, for all $(\alpha, \beta) \in[\kappa]^{2}$,

$$
c^{\prime}(\alpha, \beta):= \begin{cases}c(\alpha, \beta) & \text { if } c(\alpha, \beta)<\theta^{\prime} \\ 0 & \text { if } c(\alpha, \beta) \geq \theta^{\prime} .\end{cases}
$$

It is easily verified that $c^{\prime}$ witnesses $\mathrm{U}\left(\kappa, 2, \theta^{\prime}, \chi\right)$.

We next present some lemmas about increasing the second and fourth parameters in instances of $\mathrm{U}(\ldots)$.

Lemma 2.3. Suppose that $\mathrm{U}(\kappa, \mu, \theta, \chi)$ holds for all $\chi \in X$. If $\operatorname{cf}(\sup (X))<\operatorname{cf}(\theta)$, then $\mathrm{U}(\kappa, \mu, \theta, \sup (X))$ holds, as well.

Proof. Since $\operatorname{cf}(\sup (X))<\operatorname{cf}(\theta)$, we may assume, by thinning out $X$ if necessary, that $|X|<\operatorname{cf}(\theta)$. For each $\chi \in X$, let $c_{\chi}:[\kappa]^{2} \rightarrow \theta$ witness $\mathrm{U}(\kappa, \mu, \theta, \chi)$. Define $c:[\kappa]^{2} \rightarrow \theta$ by letting $c(\alpha, \beta):=\sup \left\{c_{\chi}(\alpha, \beta) \mid \chi \in X\right\}$ for all $(\alpha, \beta) \in[\kappa]^{2}$. It is easily verified that $c$ witnesses $\mathrm{U}(\kappa, \mu, \theta, \sup (X))$.

Lemma 2.4. Suppose that $\theta \leq \lambda$ are infinite cardinals. Then $\mathrm{U}\left(\lambda^{+}, 2, \theta, 2\right)$ holds iff $\mathrm{U}\left(\lambda^{+}, \omega, \theta, \mathrm{cf}(\lambda)\right)$ holds.

Proof. Clearly, only the forward implication needs an argument. By Corollary 4.10 below, if $\lambda$ is regular, then in fact $\mathrm{U}\left(\lambda^{+}, \lambda^{+}, \theta, \lambda\right)$ holds, so we may assume that $\lambda$ is singular. Fix a function $c:\left[\lambda^{+}\right]^{2} \rightarrow \theta$ witnessing $\mathrm{U}\left(\lambda^{+}, 2, \theta, 2\right)$. By [Rin12, Theorem 3.1], we may pick a function $t:\left[\lambda^{+}\right]^{2} \rightarrow\left[\lambda^{+}\right]^{2}$ with the property that, for every family $\mathcal{A} \subseteq\left[\lambda^{+}\right]^{<\operatorname{cf}(\lambda)}$ consisting of $\lambda^{+}$-many pairwise disjoints sets, there exists a stationary $S \subseteq \lambda^{+}$such that, for all $(\alpha, \beta) \in[S]^{2}$, there exists $(a, b) \in[\mathcal{A}]^{2}$ such that $t[a \times b]=(\alpha, \beta)$. It is easy to see that $c \circ t$ witnesses $\mathrm{U}\left(\lambda^{+}, 2, \theta, \operatorname{cf}(\lambda)\right)$, and hence, by Proposition 2.1(5), also $\mathrm{U}\left(\lambda^{+}, \omega, \theta, \operatorname{cf}(\lambda)\right)$.

Lemma 2.5. If a coloring $c:[\kappa]^{2} \rightarrow \theta$ witnesses $\mathrm{U}(\kappa, \kappa, \theta, 3)$, then it also witnesses $\mathrm{U}(\kappa, \kappa, \theta, \omega)$. 
Proof. Suppose that $c:[\kappa]^{2} \rightarrow \theta$ witnesses $\mathrm{U}(\kappa, \kappa, \theta, 3)$. We prove by induction on $n \geq 2$ that $c$ witnesses $\mathrm{U}(\kappa, \kappa, \theta, n+1)$. To this end, fix an integer $n \geq 2$, and suppose that $c$ witnesses $\mathrm{U}(\kappa, \kappa, \theta, n)$. To show that $c$ witnesses $\mathrm{U}(\kappa, \kappa, \theta, n+1)$, it suffices to show that, for every $\mathcal{A} \subseteq[\kappa]^{n}$ consisting of $\kappa$-many pairwise disjoint sets, and every $i<\theta$, there exists $\mathcal{B} \in[\mathcal{A}]^{\kappa}$ such that $\min (c[a \times b])>i$ for all $(a, b) \in[\mathcal{B}]^{2}$.

Fix such an $\mathcal{A}$ and $i$. Suppose that $\mathcal{A}$ is injectively enumerated as $\left\{a_{\alpha} \mid \alpha<\kappa\right\}$ and that, for all $\alpha<\kappa, a_{\alpha}$ is enumerated as $\left\{a_{\alpha, j} \mid j<n\right\}$. Let $\left\{p_{l} \mid l<\frac{n \cdot(n-1)}{2}\right\}$ be an injective enumeration of $\left\{\left\{j, j^{\prime}\right\} \mid j<j^{\prime}<n\right\}$. As $c$ witnesses $\mathrm{U}(\kappa, \kappa, \theta, 3)$, we may recursively find a $\subseteq$-decreasing chain $\left\langle T_{l} \mid l<\frac{n \cdot(n-1)}{2}\right\rangle$ of elements of $[\kappa]^{\kappa}$ such that for each $l$, for all distinct $\alpha, \beta$ in $T_{l}$, letting $a:=\left\{a_{\alpha, j} \mid j \in p_{l}\right\}$ and $b:=\left\{a_{\beta, j} \mid\right.$ $\left.j \in p_{l}\right\}$, if $a<b$, then $\min (c[a \times b])>i$. Now, let $\mathcal{B}:=\left\{a_{\alpha} \mid \alpha \in \bigcap_{l<\frac{n \cdot(n-1)}{2}} T_{l}\right\}$. Clearly, for every $(a, b) \in[\mathcal{B}]^{2}$, we have $\min (c[a \times b])>i$.

The preceding lemma is optimal in the following sense.

Lemma 2.6. Suppose that for some cardinal $\lambda,\left(\lambda^{<} \theta\right)^{+} \leq \kappa \leq \lambda^{\theta}$. Then there exists a coloring witnessing $\mathrm{U}(\kappa, \kappa, \theta, 2)$ that fails to witness $\mathrm{U}(\kappa, 2, \theta, 3)$.

Proof. Fix an injective enumeration $\left\{f_{\alpha} \mid \alpha<\kappa\right\}$ of some subset of ${ }^{\theta} \lambda$. Define a coloring $c:[\kappa]^{2} \rightarrow \theta$ by letting, for all $\alpha<\beta<\kappa$,

$$
c(\alpha, \beta):=\min \left\{i<\theta \mid f_{\alpha}(i) \neq f_{\beta}(i)\right\} .
$$

To see that $c$ witnesses $\mathrm{U}(\kappa, \kappa, \theta, 2)$, fix an arbitrary $\mathcal{A} \subseteq \kappa$ of size $\kappa$ and a color $i<\theta$. As $\operatorname{cf}(\kappa)=\kappa>\left|{ }^{(i+1)} \lambda\right|$, we may pick $h: i+1 \rightarrow \lambda$ for which $\mathcal{A}_{h}:=\{\alpha \in A \mid$ $\left.h \subseteq f_{\alpha}\right\}$ has size $\kappa$. Clearly, $c(\alpha, \beta)>i$ for all $(\alpha, \beta) \in\left[\mathcal{A}_{h}\right]^{2}$.

To see that $c$ does not witness $\mathrm{U}(\kappa, 2, \theta, 3)$, define $g: \operatorname{acc}(\kappa) \rightarrow \theta$ by letting $g(\alpha):=c(\alpha, \alpha+1)$ for all $\alpha \in \operatorname{acc}(\kappa)$. Pick $A \in[\operatorname{acc}(\kappa)]^{\kappa}$ on which $g$ is constant, with value, say, $i$. Clearly, for every $(\alpha, \beta) \in[A]^{2}$, if $c(\alpha, \beta)>i$, then $c(\alpha, \beta+1)=$ $c(\beta, \beta+1)=i$. It follows that $\mathcal{A}:=\{\{\alpha, \alpha+1\} \mid \alpha \in A\}$ is a family consisting of $\kappa$-many pairwise disjoint sets, and for all $(a, b) \in \mathcal{A}$, we have $\min (c[a \times b]) \leq i$.

2.1. Associated trees. In this subsection, we begin to investigate trees derived from colorings, particularly those witnessing instances of $\mathrm{U}(\ldots)$. For any coloring $c:[\kappa]^{2} \rightarrow \kappa$ and any ordinal $\gamma<\kappa$, we denote by $c(\cdot, \gamma)$ the unique function from $\gamma$ to $\kappa$ satisfying $c(\cdot, \gamma)(\alpha)=c(\alpha, \gamma)$ for all $\alpha<\gamma$. Then, the tree associated to $c$ is

$$
\mathcal{T}(c):=\{c(\cdot, \gamma) \uparrow \beta \mid \beta \leq \gamma<\kappa\} .
$$

We begin by proving that, if $c$ witnesses certain mild instances of $\mathrm{U}(\ldots)$, then $\mathcal{T}(c)$ cannot admit a cofinal branch.

Proposition 2.7. Suppose that $\theta<\kappa$ and $c:[\kappa]^{2} \rightarrow \theta$ witnesses $\mathrm{U}(\kappa, 2, \theta, 2)$. Then $\mathcal{T}(c)$ admits no cofinal branch.

Proof. Suppose not, and fix $b: \kappa \rightarrow \theta$ such that $\{b\lceil\beta \mid \beta<\kappa\} \subseteq \mathcal{T}(c)$. This means that for every ordinal $\beta<\kappa$, there exists some $\gamma \in[\beta, \kappa)$, such that $b \uparrow$ $\beta \subseteq c(\cdot, \gamma)$. Recursively construct a strictly increasing function $f: \kappa \rightarrow \kappa$ such that $b\lceil\operatorname{ssup}(f[\alpha]) \subseteq c(\cdot, f(\alpha))$ for all $\alpha<\kappa$. Write $A:=\operatorname{Im}(f)$. In particular, $c(\alpha, \gamma)=b(\alpha)$ for all $(\alpha, \gamma) \in[A]^{2}$.

As $\theta<\kappa$, let us fix $B \in[A]^{\kappa}$ on which $\alpha \mapsto b(\alpha)$ is constant with value, say, $i$. Then $\sup \left(c^{\prime \prime}[B]^{2}\right) \leq i$, contradicting the fact that $c$ witnesses $\mathrm{U}(\kappa, 2, \theta, 2)$. 
Remark 3. It is natural to ask whether the tree associated to a witness to $\mathrm{U}(\cdots)$ must be special or must be nonspecial. We shall address this question in Subsection 4.3.

Recall that a $\theta$-ascending path (resp. $\theta$-ascent path) through a $\kappa$-tree $\left(T,<_{T}\right)$ is a sequence $\left\langle f_{\alpha} \mid \alpha<\kappa\right\rangle$ such that the following two conditions hold:

- for all $\alpha<\kappa, f_{\alpha}$ is a function from $\theta$ to the $\alpha^{\text {th }}$ level of $\left(T,<_{T}\right)$;

- for all $\alpha<\beta<\kappa$, there are $j, j^{\prime}<\theta$ such that $f_{\alpha}(j)<_{T} f_{\beta}\left(j^{\prime}\right)$ (resp. $f_{\alpha}(j)<_{T} f_{\beta}(j)$ for a tail of $\left.j<\theta\right)$.

In Part III, it is proved that, if $\theta<\kappa$ and there exists a $\kappa$-tree admitting a $\theta$-ascent path but no $\theta^{\prime}$-ascent path for $\theta^{\prime}<\theta$, then $\mathrm{U}(\kappa, 2, \theta, \theta)$ holds. We now generalize Proposition 2.7 and deal with the converse of the result from Part III.

Lemma 2.8. Suppose that $\theta \in \operatorname{Reg}(\kappa), \chi<\kappa$, and $c:[\kappa]^{2} \rightarrow \theta$ witnesses $\mathrm{U}(\kappa, 2, \theta, \chi)$. For every infinite cardinal $\theta^{\prime}<\chi$,

(1) if $\theta^{\prime}<\theta$, then $\mathcal{T}(c)$ admits no $\theta^{\prime}$-ascending path;

(2) if $\operatorname{cf}\left(\theta^{\prime}\right) \neq \theta$, then $\mathcal{T}(c)$ admits no $\theta^{\prime}$-ascent path.

Proof. For each $\alpha<\kappa$, set $T_{\alpha}:=\mathcal{T}(c) \cap{ }^{\alpha} \theta$. Suppose that $\theta^{\prime}$ is an infinite cardinal less than $\chi$ and that $\left\langle f_{\alpha}: \theta^{\prime} \rightarrow T_{\alpha} \mid \alpha<\kappa\right\rangle$ is a given sequence of functions. For each $\alpha<\kappa$, fix $a_{\alpha} \in[\kappa]^{<\chi}$ such that

$$
\{c(\cdot, \alpha)\} \cup\left\{f_{\alpha+1}(j) \mid j<\theta^{\prime}\right\}=\left\{c(\cdot, \beta)\left\lceil(\alpha+1) \mid \beta \in a_{\alpha}\right\} .\right.
$$

As $\min \left(a_{\alpha}\right)=\alpha$, we may pick $A \in[\kappa]^{\kappa}$ for which $\left\{a_{\alpha} \mid \alpha \in A\right\}$ are pairwise disjoint.

(1) Suppose that $\theta^{\prime}<\theta$. For each $\alpha \in A$, let $i_{\alpha}:=\sup \left\{f_{\alpha+1}(j)(\alpha) \mid j<\theta^{\prime}\right\}$. Fix $B \in[A]^{\kappa}$ for which $\left\{i_{\alpha} \mid \alpha \in B\right\}$ is a singleton, say $\{i\}$. Pick $\left(\alpha, \alpha^{\prime}\right) \in[B]^{2}$ with $a_{\alpha}<a_{\alpha^{\prime}}$ such that $\min \left(c\left[a_{\alpha} \times a_{\alpha^{\prime}}\right]\right)>i$.

Towards a contradiction, suppose that there exist $j, j^{\prime}<\theta^{\prime}$ such that $f_{\alpha+1}(j) \subseteq$ $f_{\alpha^{\prime}+1}\left(j^{\prime}\right)$. Pick $\beta \in a_{\alpha^{\prime}}$ such that $f_{\alpha^{\prime}+1}\left(j^{\prime}\right) \subseteq c(\cdot, \beta)$. Then $c(\alpha, \beta)=f_{\alpha^{\prime}+1}\left(j^{\prime}\right)(\alpha)=$ $f_{\alpha+1}(j)(\alpha) \leq i$, contradicting the fact that $(\alpha, \beta) \in a_{\alpha} \times a_{\alpha^{\prime}}$.

(2) Suppose that $\operatorname{cf}\left(\theta^{\prime}\right) \neq \theta$. If $\operatorname{cf}\left(\theta^{\prime}\right)<\theta$ and $\mathcal{T}(c)$ admits a $\theta^{\prime}$-ascent path, then it also admits a $\operatorname{cf}\left(\theta^{\prime}\right)$-ascent path, and hence a $\operatorname{cf}\left(\theta^{\prime}\right)$-ascending path. This case is therefore covered by Clause (1).

Next, suppose that $\operatorname{cf}\left(\theta^{\prime}\right)>\theta$. For each $\alpha<\kappa$, pick $i_{\alpha}<\theta$ such that $\sup \left\{j<\theta^{\prime} \mid\right.$ $\left.f_{\alpha+1}(j)(\alpha)=i_{\alpha}\right\}=\theta^{\prime}$. Fix $B \in[A]^{\kappa}$ for which $\left\{i_{\alpha} \mid \alpha \in B\right\}$ is a singleton, say $\{i\}$. Pick $\left(\alpha, \alpha^{\prime}\right) \in[B]^{2}$ with $a_{\alpha}<a_{\alpha^{\prime}}$ such that $\min \left(c\left[a_{\alpha} \times a_{\alpha^{\prime}}\right]\right)>i$.

Towards a contradiction, suppose that $f_{\alpha+1}(j) \subseteq f_{\alpha^{\prime}+1}(j)$ for all sufficiently large $j<\theta^{\prime}$, and use this to find a $j$ for which $f_{\alpha+1}(j) \subseteq f_{\alpha^{\prime}+1}(j)$ and $f_{\alpha+1}(j)(\alpha)=$ $i_{\alpha}$. Pick $\beta \in a_{\alpha^{\prime}}$ such that $f_{\alpha^{\prime}+1}(j) \subseteq c(\cdot, \beta)$. Then $c(\alpha, \beta)=f_{\alpha^{\prime}+1}(j)(\alpha)=$ $f_{\alpha+1}(j)(\alpha)=i_{\alpha}=i$, contradicting the fact that $(\alpha, \beta) \in a_{\alpha} \times a_{\alpha^{\prime}}$.

Remark 4. In [LHL19], Lambie-Hanson and Lücke prove that, if $\kappa$ is a weakly compact cardinal and $\theta^{\prime} \in \operatorname{Reg}(\kappa)$, then, in some cofinality-preserving forcing extension, $\kappa$ remains strongly inaccessible and every $\kappa$-tree has a $\theta^{\prime}$-ascent path. It follows that, in their model, $\mathrm{U}\left(\kappa, 2, \theta,\left(\theta^{\prime}\right)^{+}\right)$fails for all $\theta \in \operatorname{Reg}(\kappa) \backslash\left\{\theta^{\prime}\right\}$. In Part II, we shall carry out a further analysis of this model, proving that it satisfies $\mathrm{U}\left(\kappa, \kappa, \theta^{\prime}, \kappa\right)$. In particular, the special case $\theta^{\prime}=\omega$ will yield a model in which $\operatorname{Pr}_{1}(\kappa, \kappa, 2,2)$ holds and $\operatorname{Pr}_{1}\left(\kappa, \kappa, 2, \omega_{1}\right)$ fails, thus showing that [Rin14, Conjecture 2] is the most one can hope for. 
Corollary 2.9. Suppose that $\kappa$ is a strongly inaccessible cardinal and $\mathrm{U}\left(\kappa, 2, \omega_{1}, \omega_{1}\right)$ holds. Then there exists a $\kappa$-Aronszajn tree with no $\omega$-ascending path.

2.2. Large cardinals. In this subsection, we indicate how large cardinals can imply nontrivial failures of $\mathrm{U}(. .$.$) at inaccessible cardinals and successors of singular$ cardinals. First, recall that a cardinal $\kappa$ is weakly compact if it is strongly inaccessible and there are no $\kappa$-Aronszajn trees. The following fact is now an immediate consequence of Proposition 2.7.

Fact 2.10. If $\kappa$ is weakly compact, then $\mathrm{U}(\kappa, 2, \theta, 2)$ fails for all $\theta \in \operatorname{Reg}(\kappa)$.

To obtain a similar result at successors of singular cardinals, we employ strongly compact cardinals. Recall that a cardinal $\nu$ is strongly compact if it is uncountable and every $\nu$-complete filter can be extended to a $\nu$-complete ultrafilter.

Theorem 2.11. Suppose that $\lambda$ is a singular limit of strongly compact cardinals. Then $\mathrm{U}\left(\lambda^{+}, 2, \theta, \operatorname{cf}(\lambda)^{+}\right)$fails for all $\theta \in \operatorname{Reg}(\lambda) \backslash\{\operatorname{cf}(\lambda)\}$.

Proof. Fix an arbitrary $\theta \in \operatorname{Reg}(\lambda) \backslash\{\operatorname{cf}(\lambda)\}$ and a coloring $c:\left[\lambda^{+}\right]^{2} \rightarrow \theta$. In order to show that $c$ does not witness $\mathrm{U}\left(\lambda^{+}, 2, \theta, \operatorname{cf}(\lambda)^{+}\right)$, we will find an $i<\theta$ and a family $\mathcal{A} \subseteq\left[\lambda^{+}\right] \leq \operatorname{cf}(\lambda)$ consisting of $\lambda^{+}$-many pairwise disjoint sets such that, for all $(a, b) \in[\mathcal{A}]^{2}$, we have $\min (c[a \times b]) \leq i$.

Let $\left\langle\lambda_{j} \mid j<\operatorname{cf}(\lambda)\right\rangle$ be an increasing sequence of strongly compact cardinals that is cofinal in $\lambda$, with $\lambda_{0}>\theta$. For a fixed $j<\operatorname{cf}(\lambda)$, use the strong compactness of $\lambda_{j}$ to pick a uniform, $\lambda_{j}$-complete ultrafilter $U_{j}$ on $\lambda^{+}$. Then, for each $\alpha<\lambda^{+}$, use the $\lambda_{j}$-completeness of $U_{j}$ to find an $i_{\alpha}^{j}<\theta$ and an $X_{\alpha}^{j} \in U_{j}$ such that for all $\beta \in X_{\alpha}^{j}$, we have $\alpha<\beta$ and $c(\alpha, \beta)=i_{\alpha}^{j}$. Then, again use the completeness of $U_{j}$ to find an $i^{j}<\theta$ and a $Y^{j} \in U_{j}$ such that, for all $\alpha \in Y^{j}$, we have $i_{\alpha}^{j}=i^{j}$.

Now, since $\theta$ is regular and $\theta \neq \operatorname{cf}(\lambda)$, we may find an $i<\theta$ and an unbounded $J \subseteq \operatorname{cf}(\lambda)$ such that, for all $j \in J$, we have $i^{j} \leq i$. For every nonzero $\delta<\lambda^{+}$, fix a sequence $\left\langle Z_{\delta}^{j} \mid j \in J\right\rangle$ such that

- $\bigcup_{j \in J} Z_{\delta}^{j}=\delta$;

- for all $j \in J, 0<\left|Z_{\delta}^{j}\right|<\lambda_{j}$.

We now construct our family $\mathcal{A}:=\left\{a_{\gamma} \mid \gamma<\lambda^{+}\right\}$by recursion on $\gamma<\lambda^{+}$. We will arrange so that, for all $\gamma<\delta<\lambda^{+}$,

- for all $j \in J$, we have $a_{\gamma} \cap Y^{j} \neq \emptyset$;

- $a_{\gamma}<a_{\delta}$

- $\min \left(c\left[a_{\gamma} \times a_{\delta}\right]\right) \leq i$.

This will clearly suffice to prove the theorem.

Begin by letting $a_{0}:=\left\{\min \left(Y^{j}\right) \mid j \in J\right\}$. Next, suppose that $\delta \in \lambda^{+} \backslash\{0\}$ and we have already constructed $\left\{a_{\gamma} \mid \gamma<\delta\right\}$. Let $\epsilon_{\delta}:=\sup \left(\bigcup_{\gamma<\delta} a_{\gamma}\right)$. For each $j \in J$ and each $\gamma \in Z_{\delta}^{j}$, fix $\alpha_{\gamma}^{j} \in a_{\gamma} \cap Y^{j}$. For each $j \in J$, use the $\lambda_{j}$-completeness of $U_{j}$ to find $\beta^{j} \in Y^{j} \cap \bigcap_{\gamma \in Z_{\delta}^{j}} X_{\alpha_{\gamma}^{j}}^{j}$ with $\beta^{j}>\epsilon_{\delta}$. Let $a_{\delta}:=\left\{\beta^{j} \mid j \in J\right\}$.

It remains to check that we have maintained the recursion hypotheses. We clearly have both $a_{\delta} \cap Y^{j} \neq \emptyset$ for all $j \in J$, and also $a_{\gamma}<a_{\delta}$ for all $\gamma<\delta$. To see that $\min \left(c\left[a_{\gamma} \times a_{\delta}\right]\right) \leq i$ for all $\gamma<\delta$, fix such a $\gamma$ and fix $j \in J$ such that $\gamma \in Z_{\delta}^{j}$. Then $\alpha_{\gamma}^{j} \in a_{\gamma} \cap Y^{j}$ and $\beta^{j} \in a_{\gamma} \cap X_{\alpha_{\gamma}^{j}}^{j}$, so $c\left(\alpha_{\gamma}^{j}, \beta^{j}\right)=i^{j} \leq i$, and we are done.

Remark 5. Let us note here two ways in which the preceding result is optimal. First, by Corollary 4.13 below, $\mathrm{U}\left(\lambda^{+}, \lambda^{+}, \operatorname{cf}(\lambda), \lambda\right)$ holds for every singular cardinal $\lambda$, so 
the requirement " $\theta \neq \operatorname{cf}(\lambda)$ " cannot be waived. Second, recall that $\mathrm{SCH}$ holds above a strongly compact cardinal. In particular, in the setting of the preceding result, we have $2^{\lambda}=\lambda^{+}$. It then follows from Theorem 4.15 below that $\mathrm{U}\left(\lambda^{+}, \lambda^{+}, \theta, \operatorname{cf}(\lambda)\right)$ holds for all $\theta<\lambda$, so the fourth parameter cannot be reduced from $\operatorname{cf}(\lambda)^{+}$.

In Part II, we will force over models with large cardinals to obtain a finer separation between instances of $\mathrm{U}(\ldots)$. In particular, we will obtain the following consistency results.

- We will force with a cofinality-preserving forcing notion over a model in which $\kappa$ is weakly compact and $\theta<\kappa$ is regular to obtain a model in which $\mathrm{U}(\kappa, \kappa, \theta, \kappa)$ holds but $\mathrm{U}\left(\kappa, 2, \theta^{\prime}, \theta^{+}\right)$fails for every $\theta^{\prime} \in \operatorname{Reg}(\kappa) \backslash\{\theta\}$.

- We will force with a cofinality-preserving forcing notion over a model in which $\lambda$ is a singular limit of supercompact cardinals and $\theta$ is a regular cardinal with $\operatorname{cf}(\lambda) \leq \theta<\lambda$ to obtain a model in which $\mathrm{U}\left(\lambda^{+}, \lambda^{+}, \theta, \lambda\right)$ holds but $\mathrm{U}\left(\lambda^{+}, 2, \theta^{\prime}, \theta^{+}\right)$fails for all $\theta^{\prime} \in \operatorname{Reg}(\lambda) \backslash\{\operatorname{cf}(\lambda), \theta\}$.

\section{STRONG CHAIN CONDITIONS AND FORCING AXIOMS}

The work in this paper arose in part from questions regarding the infinite productivity of chain conditions and possible generalizations of Martin's Axiom to higher cardinals. In this section, we present these questions and indicate how the property $\mathrm{U}(\kappa, \mu, \theta, \chi)$ comes to bear on them.

3.1. Infinite productivity of strong chain conditions. We start this section by recalling some relevant properties of posets, starting with closure properties.

Definition 3.1. Let $\mathbb{P}$ be a poset and $\lambda$ be a regular, uncountable cardinal.

(1) $\mathbb{P}$ is well-met if, whenever $p, q \in \mathbb{P}$ are compatible, they have a greatest lower bound in $\mathbb{P}$.

(2) $\mathbb{P}$ is $\lambda$-closed (resp. $\lambda$-directed closed) with greatest lower bounds if, whenever $\tau<\lambda$ and $\left\langle q_{\eta} \mid \eta<\tau\right\rangle$ is $\leq_{\mathbb{P}}$-decreasing (resp. $\leq_{\mathbb{P}}$-directed), it has a greatest lower bound in $\mathbb{P}$.

We next recall some strengthenings of the $\kappa$-chain condition.

Definition 3.2. Let $\mathbb{P}$ be a poset.

(1) A subset $B \subseteq \mathbb{P}$ is linked if it consists of pairwise compatible conditions. $B$ is centered if every finite subset of $B$ has a lower bound in $\mathbb{P}$.

(2) $\mathbb{P}$ is $\kappa$-Knaster if, whenever $A \in[\mathbb{P}]^{\kappa}$, there is $B \in[A]^{\kappa}$ that is linked.

(3) $\mathbb{P}$ has precaliber $\kappa$ if, whenever $A \in[\mathbb{P}]^{\kappa}$, there is $B \in[A]^{\kappa}$ that is centered.

(4) For a cardinal $\lambda, \mathbb{P}$ is $\lambda$-centered (resp. $\lambda$-linked) if there is a collection of $\lambda$-many centered (resp. linked) subsets of $\mathbb{P}$ that covers $\mathbb{P}$. Note that, if $\lambda<\kappa$ and $\mathbb{P}$ is $\lambda$-centered or $\lambda$-linked, then $\mathbb{P}$ has the $\kappa$-cc.

One nice feature of these strong chain conditions is the fact that they are productive, i.e., if $\mathbb{P}$ and $\mathbb{Q}$ are $\kappa$-Knaster (or have precaliber $\kappa$ or are $\lambda$-linked or $\lambda$ centered), then $\mathbb{P} \times \mathbb{Q}$ is $\kappa$-Knaster (or has precaliber $\kappa$ or is $\lambda$-linked or $\lambda$-centered, respectively). This is in contrast to the $\kappa$-cc, which is not in general productive.

It is natural to investigate the extent to which these chain conditions can be more than finitely productive. Note that, if $\theta<\kappa$ and $\mathbb{P}_{\eta}$ has the $\kappa$-cc (is $\kappa$-Knaster or has precaliber $\kappa$, resp.) for all $\eta<\theta$, then the lottery sum $\mathbb{P}:=\bigoplus_{\eta<\theta} \mathbb{P}_{\eta}$ also has the $\kappa$-cc (is $\kappa$-Knaster or has precaliber $\kappa$, resp.), so questions about the productivity of 
these conditions reduce to questions about powers of forcing posets. In particular, for regular cardinals $\theta<\kappa$, we are interested in the following question: If $\mathbb{P}$ is a poset such that $\mathbb{P}^{\tau}$ is $\kappa$-Knaster (resp. has precaliber $\kappa$ ) for all $\tau<\theta$, does it follow that $\mathbb{P}^{\theta}$ is $\kappa$-Knaster (resp. has precaliber $\kappa$ ). Note that, if $\theta=\aleph_{0}$, this is simply asking whether being $\kappa$-Knaster (resp. having precaliber $\kappa$ ) is countably productive. One can also ask for weaker conclusions, e.g., if $\mathbb{P}^{\tau}$ is $\kappa$-Knaster for all $\tau<\theta$, does it follow that $\mathbb{P}^{\theta}$ has the $\kappa$-cc?

If $\kappa$ is a weakly compact cardinal, then a poset has the $\kappa$-cc if and only if it is $\kappa$-Knaster, and both of these properties are $\theta$-productive for all $\theta<\kappa$ (i.e., if $\mathbb{P}$ is $\kappa$-Knaster, then $\mathbb{P}^{\theta}$ is also $\kappa$-Knaster). In [CL17], Cox and Lücke show that, relative to the consistency of a weakly compact cardinal, it is consistent that there is a strongly inaccessible, non-weakly-compact cardinal $\kappa$ such that the $\kappa$-Knaster property is $\theta$-productive for all $\theta<\kappa$. On the other hand, the first author and Lücke show in [LHL19] that, if $\kappa$ is a regular uncountable cardinal and the $\kappa$-Knaster property is infinitely productive, then $\kappa$ is weakly compact in L. The question as to whether the $\kappa$-Knaster property can consistently be infinitely productive when $\kappa$ is a successor cardinal (in particular, when $\kappa=\aleph_{2}$ ) is raised but left unanswered in [LHL19]. It is resolved negatively by the following lemma, together with the fact (see Corollary 4.8) that $\mathrm{U}\left(\lambda^{+}, \lambda^{+}, \omega, \omega\right)$ holds for every infinite cardinal $\lambda$.

Lemma 3.3. Suppose that $\chi, \theta \in \operatorname{Reg}(\kappa)$ and that $\kappa$ is $(<\chi)$-inaccessible. For every coloring $c:[\kappa]^{2} \rightarrow \theta$ witnessing $\mathrm{U}(\kappa, \mu, \theta, \chi)$, there exists a corresponding poset $\mathbb{P}$ such that

(1) $\mathbb{P}$ is well-met and $\chi$-directed closed with greatest lower bounds;

(2) if $\mu=2$, then $\mathbb{P}^{\tau}$ is $\kappa$-cc for all $\tau<\min (\{\chi, \theta\})$;

(3) if $\mu=\kappa$, then $\mathbb{P}^{\tau}$ has precaliber $\kappa$ for all $\tau<\min (\{\chi, \theta\})$;

(4) $\mathbb{P}^{\theta}$ is not $\kappa$-cc.

Proof. This is a straightforward variation of the proof that $\operatorname{Pr}_{1}(\ldots)$ entails counterexamples to productivity of the chain condition. Let $c:[\kappa]^{2} \rightarrow \theta$ witness $\mathrm{U}(\kappa, \mu, \theta, \chi)$, and let

$$
\mathbb{P}:=\left\{(i, x) \mid i<\theta, x \in[\kappa]^{<\chi},\left(c^{*}[x]^{2}\right) \cap i=\emptyset\right\} \cup\{\mathbf{1}\},
$$

with $(i, x) \leq_{\mathbb{P}}(j, y)$ iff $i=j$ and $x \supseteq y$, and with $(i, x) \leq_{\mathbb{P}} \mathbf{1}$ for all $(i, x)$. For ease of notation, for each $p=(i, x) \in \mathbb{P}$, we let $i_{p}$ denote $i$ and $x_{p}$ denote $x$. Clearly, $\mathbb{P}$ is well-met and $\chi$-directed closed with greatest lower bounds.

Claim 3.3.1. $\mathbb{P}^{\theta}$ has an antichain of size $\kappa$.

Proof. We shall prove a slightly stronger result. Define the support, $\operatorname{supp}(q)$, of a condition $q \in \mathbb{P}^{\theta}$, by letting $\operatorname{supp}(q):=\{j<\theta \mid q(j) \neq \mathbf{1}\}$. Let $J \in[\theta]^{\theta}$ be arbitrary. We now prove that $\left\{q \in \mathbb{P}^{\theta} \mid \operatorname{supp}(q)=J\right\}$ has an antichain of size $\kappa$. For each $\alpha<\kappa$, define an element $q_{\alpha}$ in $\mathbb{P}^{\theta}$ by letting for all $j<\theta$ :

$$
q_{\alpha}(j):= \begin{cases}(j,\{\alpha\}) & \text { if } j \in J \\ \mathbf{1} & \text { if } j \notin J .\end{cases}
$$

Then, for any pair $(\alpha, \beta) \in[\kappa]^{2}$, we get that $q_{\alpha}(j)$ and $q_{\beta}(j)$ are incompatible in $\mathbb{P}$ for $j:=\min (J \backslash c(\alpha, \beta))$. Consequently, $\left\{q_{\alpha} \mid \alpha<\kappa\right\}$ is an antichain in $\left\{q \in \mathbb{P}^{\theta} \mid\right.$ $\operatorname{supp}(q)=J\}$. 
Next, to prove Clauses (2) and (3), let $\tau<\min (\{\chi, \theta\})$, and let $A$ be an arbitrary $\kappa$-sized subset of $\mathbb{P}^{\tau}$. Without loss of generality, $A \subseteq(\mathbb{P} \backslash\{\mathbf{1}\})^{\tau}$.

For each $q: \tau \rightarrow \mathbb{P}$ in $A$, let $i^{q}:=\left\langle i_{q(j)} \mid j<\tau\right\rangle$ and $x^{q}:=\bigcup\left\{x_{q(j)} \mid j<\tau\right\}$. Since $\tau<\min (\{\chi, \theta\})$, and by the regularity of $\chi$ and $\theta$, we have $\sup \left(\operatorname{Im}\left(i^{q}\right)\right)<\theta$ and $x^{q} \in[\kappa]^{<\chi}$ for all $q \in A$. Using the fact that $\kappa$ is $(<\chi)$-inaccessible, fix $A^{\prime} \in[A]^{\kappa}$ such that

- $\left\{i^{q} \mid q \in A^{\prime}\right\}$ is a singleton, say, $\left\{i^{*}\right\}$;

- $\left\{x^{q} \mid q \in A^{\prime}\right\}$ forms a head-tail-tail $\Delta$-system, with root, say, $r$;

- $q \mapsto\left\langle x_{q(j)} \cap r \mid j<\tau\right\rangle$ is constant over $A^{\prime}$.

Let $\mathcal{A}:=\left\{x^{q} \backslash r \mid q \in A^{\prime}\right\}$. Since $c$ witnesses $\mathrm{U}(\kappa, \mu, \theta, \chi)$, we may find $\mathcal{B} \in[\mathcal{A}]^{\mu}$ such that, for all $\left(b, b^{\prime}\right) \in[\mathcal{B}]^{2}$, we have $\min \left(c\left[b \times b^{\prime}\right]\right)>\sup \left(i^{*}\right)$. Fix $B \in\left[A^{\prime}\right]^{\mu}$ such that $\left\{x^{q} \backslash r \mid q \in B\right\}=\mathcal{B}$. We claim that $B$ is centered.

Fix a finite subset $\left\{q_{m} \mid m<n\right\}$ of $B$. Define a function $q$ with domain $\tau$ by letting $q(j):=\left(i^{*}(j), \bigcup_{m<n} x_{q_{m}(j)}\right)$ for all $j<\tau$. To prove that $q$ is a lower bound for $\left\{q_{m} \mid m<n\right\}$, it suffices to verify that $q \in \mathbb{P}^{\tau}$. If not, then there are $m, m^{\prime}<n$, $j<\tau$, and $\alpha<\alpha^{\prime}<\kappa$ such that

- $\alpha \in x_{q_{m}(j)} \backslash x_{q_{m^{\prime}}(j)}$;

- $\alpha^{\prime} \in x_{q_{m^{\prime}}(j)} \backslash x_{q_{m}(j)}$;

- $c\left(\alpha, \alpha^{\prime}\right)<i^{*}(j)$.

Since $x_{q_{m}(j)} \cap r=x_{q_{m^{\prime}}(j)} \cap r$, it follows that $\alpha \in x_{q_{m}(j)} \backslash r$ and $\alpha^{\prime} \in x_{q_{m^{\prime}}(j)} \backslash r$. But $x_{q_{m}(j)} \backslash r, x_{q_{m^{\prime}}(j)} \backslash r \in \mathcal{B}$, so we have $c\left(\alpha, \alpha^{\prime}\right)>\sup \left(\operatorname{Im}\left(i^{*}\right)\right) \geq i^{*}(j)$. Thus, $B$ is centered, as desired.

Corollary 3.4. If $\kappa$ is a regular uncountable cardinal and $\mathrm{U}(\kappa, \kappa, \omega, \omega)$ holds, then there exists a $\kappa$-Knaster poset $\mathbb{P}$ such that $\mathbb{P}^{\omega}$ is not $\kappa$-cc.

3.2. Forcing axioms. Beginning in the 1970s, much work has been done attempting to generalize Martin's Axiom to higher cardinals, and to $\aleph_{2}$ in particular. Versions of such a generalization were obtained in unpublished work of both Laver and Baumgartner, and a stronger version was obtained by Shelah in [She78]. We state here the version due to Baumgartner. We denote the axiom by BA; more information regarding BA can be found in [Tal94].

Definition 3.5. Let $\mathbb{P}$ be a forcing poset, and let $\nu$ be a cardinal. $\mathrm{FA}_{\nu}(\mathbb{P})$ is the assertion that, whenever $\left\{D_{\alpha} \mid \alpha<\nu\right\}$ is a collection of dense subsets of $\mathbb{P}$, then there is a filter $G \subseteq \mathbb{P}$ such that, for all $\alpha<\nu, G \cap D_{\alpha} \neq \emptyset$.

Definition 3.6 (Baumgartner's Axiom). BA is the statement that, if $\mathbb{P}$ is a poset that is well-met, countably closed, and $\aleph_{1}$-linked, then $\mathrm{FA}_{\nu}(\mathbb{P})$ holds for all $\nu<2^{\aleph_{1}}$.

Fact 3.7 (Baumgartner). Suppose that $\mathrm{CH}$ holds and $\kappa \geq \aleph_{2}$ is regular. Then there is a cofinality-preserving forcing extension in which $\mathrm{BA}+\mathrm{CH}+2^{\aleph_{1}}=\kappa$ holds.

Shelah and Stanley, in [SS82], prove that Fact 3.7 fails if BA is weakened by omitting the requirement that $\mathbb{P}$ be well-met. In particular, they prove the following result. (They prove the result for $\lambda=\aleph_{1}$, but their proof generalizes.)

Fact 3.8 (Shelah-Stanley, [SS82]). Suppose that $\lambda$ is an uncountable cardinal and $\lambda^{<\lambda}=\lambda$. Then there is a poset $\mathbb{P}$ of size $\lambda^{+}$that is $\lambda$-closed and $\lambda$-linked but for which $\mathrm{FA}_{\lambda^{+}}(\mathbb{P})$ fails. 
The work in this paper was partially motivated by the following unpublished result of Inamdar, which indicates another way in which BA cannot consistently be changed. In particular, the requirement that $\mathbb{P}$ be $\aleph_{1}$-linked cannot be replaced by the requirement that $\mathbb{P}$ has precaliber $\aleph_{2}$. We would like to thank Inamdar for allowing us to include this theorem.

Theorem 3.9 (Inamdar, [Ina17]). Suppose that $\lambda=\lambda^{<\lambda}$ is a regular uncountable cardinal. Then there is a forcing poset $\mathbb{Q}$ of size $\lambda^{+}$such that

(1) $\mathbb{Q}$ is well-met and $\lambda$-directed closed with greatest lower bounds;

(2) $\mathbb{Q}$ has precaliber $\lambda^{+}$;

(3) $\mathrm{FA}_{\lambda^{+}}(\mathbb{Q})$ fails.

Proof. By Corollary 4.8 (see also Corollary 4.10), we can fix a function $c:\left[\lambda^{+}\right]^{2} \rightarrow \omega$ witnessing $\mathrm{U}\left(\lambda^{+}, \lambda^{+}, \omega, \lambda\right)$. For each $i<\omega$, define a poset $\mathbb{P}_{i}:=\left\{x \in\left[\lambda^{+}\right]^{<\lambda} \mid\right.$ $\left.c^{\text {" }}[x]^{2} \cap i=\emptyset\right\}$, ordered by reverse inclusion, and let $\mathbb{Q}_{i}$ be the $<\lambda$-support product of $\lambda$ copies of $\mathbb{P}_{i}$. It is immediate that, for all $i<\omega, \mathbb{Q}_{i}$ has size $\lambda^{+}$and is well-met and $\lambda$-directed closed with greatest lower bounds.

Claim 3.9.1. For all $i<\omega, \mathbb{Q}_{i}$ has precaliber $\lambda^{+}$.

Proof. The proof is essentially the same as that of Clause (3) of Lemma 3.3.

Claim 3.9.2. Suppose that $i<\omega$ and $\mathrm{FA}_{\lambda^{+}}\left(\mathbb{Q}_{i}\right)$ holds. Then $\mathbb{P}_{i}$ is $\lambda$-centered.

Proof. For each $p \in \mathbb{P}_{i}$, the set $D_{p}:=\left\{q \in \mathbb{Q}_{i} \mid\right.$ for some $\left.\eta \in \operatorname{dom}(q), q(\eta) \leq_{\mathbb{P}_{i}} p\right\}$ is dense in $\mathbb{Q}_{i}$. As $\left|\mathbb{P}_{i}\right|=\lambda^{+}$, there is a filter $G \subseteq \mathbb{Q}_{i}$ such that $G \cap D_{p} \neq \emptyset$ for all $p \in \mathbb{P}_{i}$. For $\eta<\lambda$, let $G_{\eta}$ be the upward closure of $\{q(\eta) \mid q \in G\}$ in $\mathbb{P}_{i}$. Then each $G_{\eta}$ is a centered subset of $\mathbb{P}_{i}$, and $\bigcup_{\eta<\lambda} G_{\eta}=\mathbb{P}_{i}$, so $\mathbb{P}_{i}$ is $\lambda$-centered.

Claim 3.9.3. Suppose that $\mathbb{P}_{i}$ is $\lambda$-centered for all $i<\omega$. Then $\prod_{i<\omega} \mathbb{P}_{i}$ is $\lambda$ centered.

Proof. For each $i<\omega$, let $\left\{G_{\eta}^{i} \mid \eta<\lambda\right\}$ be a collection of centered subsets that covers $\mathbb{P}_{i}$. For each $h \in{ }^{\omega} \lambda$, let

$$
G_{h}:=\left\{p \in \prod_{i<\omega} \mathbb{P}_{i} \mid \text { for all } i<\omega, p(i) \in G_{h(i)}^{i}\right\} .
$$

Then $\left\{G_{h} \mid h \in{ }^{\omega} \lambda\right\}$ is a collection of centered subsets that covers $\mathbb{P}_{i}$. Since $\lambda^{\aleph_{0}}=\lambda$, the collection has size $\lambda$ and hence witnesses that $\prod_{i<\omega} \mathbb{P}_{i}$ is $\lambda$-centered.

Therefore, if $\mathrm{FA}_{\lambda^{+}}\left(\mathbb{Q}_{i}\right)$ holds for all $i<\omega$, then $\prod_{i<\omega} \mathbb{P}_{i}$ is $\lambda$-centered and hence has the $\lambda^{+}$-cc. However, if, for all $\alpha<\lambda^{+}$, we let $p_{\alpha} \in \prod_{i<\omega} \mathbb{P}_{i}$ be the constant function taking value $\{\alpha\}$, then, as in the proof of Lemma 3.3, $\left\{p_{\alpha} \mid \alpha<\lambda^{+}\right\}$is an antichain of size $\lambda^{+}$in $\prod_{i<\omega} \mathbb{P}_{i}$. It follows that there is $i<\omega$ for which $\mathrm{FA}_{\lambda^{+}}\left(\mathbb{Q}_{i}\right)$ fails.

\section{Closed COlorings}

4.1. Preliminaries. In this section, we undertake a thorough analysis of witnesses to $\mathrm{U}(\kappa, \mu, \theta, \chi)$ that satisfy certain closure conditions isolated by the following definition, and the circumstances under which such colorings must exist. Our reasons for focusing on closed colorings are twofold. Firstly, closed colorings behave more nicely than general colorings. For example, as Lemma 4.2 will make clear, a closed 
witness to $\mathrm{U}(\kappa, 2, \theta, \chi)$ is actually a witness to $\mathrm{U}(\kappa, \kappa, \theta, \chi)$. Secondly, closed colorings seem to arise naturally. Our primary methods for constructing witnesses to $\mathrm{U}(\kappa, \mu, \theta, \chi)$ come from the techniques of walks on ordinals, and, as we shall see in this section, the colorings that arise from these constructions tend to be closed.

Definition 4.1. For a subset $\Sigma \subseteq \kappa$, we say that $c:[\kappa]^{2} \rightarrow \theta$ is $\Sigma$-closed if, for all $\beta<\kappa$ and $i \leq \theta$, the set $D_{\leq i}^{c}(\beta):=\{\alpha<\beta \mid c(\alpha, \beta) \leq i\}$ satisfies $\operatorname{acc}^{+}\left(D_{\leq i}^{c}(\beta)\right) \cap \Sigma \subseteq D_{\leq i}^{c}(\beta)$. We say that $c$ is somewhere-closed iff it is $\Sigma$-closed for some stationary $\Sigma \subseteq \kappa$, that $c$ is tail-closed iff it is $E_{\geq \sigma}^{\kappa}$-closed for some $\sigma \in \operatorname{Reg}(\kappa)$, and that $c$ is closed iff it is $\kappa$-closed.

Lemma 4.2. Suppose that $c:[\kappa]^{2} \rightarrow \theta$ is a coloring and $\omega \leq \chi<\kappa$. Then $(1) \Longrightarrow(2) \Longrightarrow(3)$ :

(1) For some stationary $\Sigma \subseteq E_{\geq \chi}^{\kappa}, c$ is a $\Sigma$-closed witness to $\mathrm{U}(\kappa, 2, \theta, \chi)$.

(2) For every family $\mathcal{A} \subseteq[\kappa]^{<\chi}$ consisting of $\kappa$-many pairwise disjoint sets, for every club $D \subseteq \kappa$, and for every $i<\theta$, there exist $\gamma \in D, a \in \mathcal{A}$, and $\epsilon<\gamma$ such that:

- $\gamma<a$;

- for all $\alpha \in(\epsilon, \gamma)$ and all $\beta \in a$, we have $c(\alpha, \beta)>i$.

(3) c witnesses $\mathrm{U}(\kappa, \kappa, \theta, \chi)$.

Proof. (1) $\Longrightarrow$ (2) Fix a family $\mathcal{A} \subseteq[\kappa]<\chi$ consisting of $\kappa$-many pairwise disjoint sets, a club $D \subseteq \kappa$, and a color $i<\theta$. Find $\mathcal{X} \subseteq[\kappa]^{<\chi}$ consisting of $\kappa$-many pairwise disjoint sets such that every $x \in \mathcal{X}$ is of the form $\{\gamma\} \cup a$ for some $\gamma \in \Sigma \cap D$ and $a \in \mathcal{A}$. As $c$ witnesses $\mathrm{U}(\kappa, 2, \theta, \chi)$, we may pick $(x, y) \in[\mathcal{X}]^{2}$ such that $\min (c[x \times y])>i$. Fix $\gamma \in(\Sigma \cap D) \cap x$ and $a \in \mathcal{A} \cap \mathcal{P}(y)$. Clearly, $\gamma<a$ and $|a|<\chi \leq \operatorname{cf}(\gamma)$. Now, let $\beta \in a$ be arbitrary. Since $(\gamma, \beta) \in x \times y$, we have $c(\gamma, \beta)>i$, and, since $\gamma \in \Sigma$, there must exist $\epsilon(\gamma, \beta)<\gamma$ such that, for all $\alpha \in(\epsilon(\gamma, \beta), \gamma), c(\alpha, \beta)>i$. Since $\operatorname{cf}(\gamma) \geq \chi>|a|$, we have that $\epsilon:=\sup \{\epsilon(\gamma, \beta) \mid$ $\beta \in a\}$ is less than $\gamma$. Then $\gamma, a$, and $\epsilon$ are as sought.

$(2) \Longrightarrow(3)$ Fix a family $\mathcal{A} \subseteq[\kappa]^{<\chi}$ consisting of $\kappa$-many pairwise disjoint sets and a color $i<\theta$. Let $\Gamma$ denote the collection of all $\gamma<\kappa$ such that for some $a_{\gamma} \in \mathcal{A}$ and some $\epsilon_{\gamma}<\gamma$, we have

- $\gamma<a_{\gamma}$; and

- for all $\alpha \in\left(\epsilon_{\gamma}, \gamma\right)$ and all $\beta \in a_{\gamma}$, we have $c(\alpha, \beta)>i$.

By the hypothesis, $\Gamma$ is stationary. Define $f: \Gamma \rightarrow \kappa$ and $g: \Gamma \rightarrow \kappa$ by letting, for all $\gamma \in \Gamma, f(\gamma):=\epsilon_{\gamma}$ and $g(\gamma):=\sup \left(a_{\gamma}\right)$. By Fodor's Lemma, we now pick $\epsilon<\kappa$ for which $B:=\{\gamma \in \Gamma \mid f(\gamma)=\epsilon \& g[\gamma] \subseteq \gamma\}$ is stationary, and then let $\mathcal{B}:=\left\{a_{\gamma} \mid \gamma \in B\right\}$. We claim that $\min (c[a \times b])>i$ for all $(a, b) \in[\mathcal{B}]^{2}$. To this end, fix $(a, b) \in[\mathcal{B}]^{2}$, and let $\gamma, \delta \in B$ be such that $a=a_{\gamma}$ and $b=a_{\delta}$. Then we have

and hence $c(\alpha, \beta)>i$.

$$
\epsilon_{\delta}=\epsilon<\gamma<\alpha<\delta<\beta
$$

4.2. Walks on ordinals. We now introduce some of the machinery we will need to construct witnesses to $\mathrm{U}(\kappa, \mu, \theta, \chi)$ using the techniques of walks on ordinals.

Definition 4.3. A $C$-sequence over $\kappa$ is a sequence $\left\langle C_{\alpha} \mid \alpha<\kappa\right\rangle$ such that, for all $\alpha<\kappa$,

- $C_{0}=\emptyset$; 
- $C_{\alpha+1}=\{\alpha\}$

- if $\alpha \in \operatorname{acc}(\kappa)$, then $C_{\alpha}$ is a club in $\alpha$.

Definition 4.4 ([Tod87]). Given a $C$-sequence $\left\langle C_{\alpha} \mid \alpha<\kappa\right\rangle$, we derive various functions as follows. For all $\alpha<\beta<\kappa$,

- $\operatorname{Tr}(\alpha, \beta) \in{ }^{\omega} \kappa$ is defined recursively by letting, for all $n<\omega$,

$\operatorname{Tr}(\alpha, \beta)(n):= \begin{cases}\beta & \text { if } n=0 ; \\ \min \left(C_{\operatorname{Tr}(\alpha, \beta)(n-1)} \backslash \alpha\right) & \text { if } n>0 \& \operatorname{Tr}(\alpha, \beta)(n-1)>\alpha ; \\ \alpha & \text { otherwise; }\end{cases}$

- (Number of steps) $\rho_{2}(\alpha, \beta):=\min \{n<\omega \mid \operatorname{Tr}(\alpha, \beta)(n)=\alpha\}$;

- (Upper trace) $\operatorname{tr}(\alpha, \beta):=\operatorname{Tr}(\alpha, \beta)\left\lceil\rho_{2}(\alpha, \beta)\right.$.

Remark 6 . To avoid notational confusion, note that there is no relationship between the two-place instance $\operatorname{Tr}(\alpha, \beta)$ and the one-place instance $\operatorname{Tr}(S)$.

Definition 4.5 ([Rin14]). For $\alpha<\beta<\kappa$, let

$$
\lambda_{2}(\alpha, \beta):=\sup \left(\alpha \cap\left\{\sup \left(C_{\delta} \cap \alpha\right) \mid \delta \in \operatorname{Im}(\operatorname{tr}(\alpha, \beta))\right\}\right) .
$$

Note that $\lambda_{2}(\alpha, \beta)<\alpha$ whenever $0<\alpha<\beta<\kappa$. To motivate the preceding definition, let us point out the following lemma.

Lemma 4.6. Suppose that $\lambda_{2}(\gamma, \beta)<\alpha<\gamma<\beta<\kappa$. Then $\operatorname{tr}(\gamma, \beta) \sqsubseteq \operatorname{tr}(\alpha, \beta)$ and one of the following cases holds:

(1) $\gamma \in \operatorname{Im}(\operatorname{tr}(\alpha, \beta))$; or

(2) $\gamma \in \operatorname{acc}\left(C_{\delta}\right)$ for $\delta:=\min (\operatorname{Im}(\operatorname{tr}(\gamma, \beta)))$. In particular, $\gamma \in \operatorname{acc}\left(C_{\delta}\right)$ for some $\delta \in \operatorname{Im}(\operatorname{tr}(\alpha, \beta))$.

Proof. We first show, by induction on $i$, that $\operatorname{Tr}(\alpha, \beta)(i)=\operatorname{Tr}(\gamma, \beta)(i)$ for all $i<$ $\rho_{2}(\gamma, \beta)$, i.e., that $\operatorname{tr}(\gamma, \beta) \sqsubseteq \operatorname{tr}(\alpha, \beta)$. Clearly, $\operatorname{Tr}(\alpha, \beta)(0)=\beta=\operatorname{Tr}(\gamma, \beta)(0)$. Next, suppose that $i+1<\rho_{2}(\gamma, \beta)$ and $\operatorname{Tr}(\alpha, \beta)(i)=\operatorname{Tr}(\gamma, \beta)(i)$. Since $i+1<\rho_{2}(\gamma, \beta)$, it must be the case that $\gamma \notin C_{\operatorname{Tr}(\gamma, \beta)(i)}$, and therefore $\sup \left(C_{\operatorname{Tr}(\gamma, \beta)(i)} \cap \gamma\right) \leq \lambda_{2}(\gamma, \beta)<$ $\alpha$. It follows that

$$
\min \left(C_{\operatorname{Tr}(\alpha, \beta)(i)} \backslash \alpha\right)=\min \left(C_{\operatorname{Tr}(\gamma, \beta)(i)} \backslash \alpha\right)=\min \left(C_{\operatorname{Tr}(\gamma, \beta)(i)} \backslash \gamma\right),
$$

and hence $\operatorname{Tr}(\alpha, \beta)(i+1)=\operatorname{Tr}(\gamma, \beta)(i+1)$.

To prove the second part of the lemma, set $n:=\rho_{2}(\gamma, \beta)-1$ and $\delta:=\operatorname{tr}(\gamma, \beta)(n)=$ $\operatorname{tr}(\alpha, \beta)(n)$, note that $\gamma \in C_{\delta}$, and consider the following two cases.

- If $\gamma \in \operatorname{nacc}\left(C_{\delta}\right)$, then $\sup \left(C_{\delta} \cap \gamma\right) \leq \lambda_{2}(\gamma, \beta)<\alpha$, so $\gamma=\operatorname{tr}(\alpha, \beta)(n+1)$ and we are in Case (1) of the statement of the lemma.

- If $\gamma \in \operatorname{acc}\left(C_{\delta}\right)$, then we are in Case (2) of the statement of the lemma.

Corollary 4.7. $\rho_{2}:[\kappa]^{2} \rightarrow \omega$ is closed.

Proof. Fix $\beta<\kappa, i<\omega$, and $A \subseteq D_{\leq i}^{\rho_{2}}(\beta)$ with $\gamma:=\sup (A)$ smaller than $\beta$. Fix $\alpha \in A$ above $\lambda_{2}(\gamma, \beta)$. Then, by Lemma 4.6, $\rho_{2}(\gamma, \beta) \leq \rho_{2}(\alpha, \beta) \leq i$, so $\gamma \in D_{\leq i}^{\rho_{2}}(\beta)$.

The following corollary now follows from [Tod07, Theorem 6.3.6].

Corollary 4.8 (Todorcevic). For every infinite cardinal $\lambda$, there is a closed witness to $\mathrm{U}\left(\lambda^{+}, \lambda^{+}, \omega, \operatorname{cf}(\lambda)\right)$. 
Remark 7. The statement of [Tod07, Theorem 6.3.6] in that source has a typing error, where "of size $<\kappa$ " should have been "of size $<\operatorname{cf}(\kappa)$ ". For example, by Theorem 2.11 above, if $\lambda$ is a singular limit of strongly compact cardinals and $\lambda$ has uncountable cofinality, then $\mathrm{U}\left(\lambda^{+}, \lambda^{+}, \omega, \operatorname{cf}(\lambda)^{+}\right)$fails.

4.3. The first construction. We are now ready to begin constructing closed witnesses to $\mathrm{U}(\kappa, \mu, \theta, \chi)$ using walks on ordinals. Our first result shows that the existence of closed witnesses to $\mathrm{U}(\kappa, \kappa, \theta, \chi)$ follows from the existence of certain strong counterexamples to $\operatorname{Refl}\left(\theta, E_{\geq \chi}^{\kappa}\right)$.

Theorem 4.9. Suppose that $\theta \in \operatorname{Reg}(\kappa)$ and there exist a sequence $\left\langle H_{i} \mid i<\theta\right\rangle$ of pairwise disjoint subsets of $\kappa$ and a $C$-sequence $\vec{C}=\left\langle C_{\alpha} \mid \alpha<\kappa\right\rangle$ such that, for every $\alpha \in \operatorname{acc}(\kappa)$,

$$
\sup \left\{i<\theta \mid \operatorname{acc}\left(C_{\alpha}\right) \cap H_{i} \neq \emptyset\right\}<\theta .
$$

Then there exists a closed coloring $c:[\kappa]^{2} \rightarrow \theta$ such that

(1) for every $\chi \in \operatorname{Reg}(\kappa)$ for which

$$
\sup \left\{i<\theta \mid E_{\geq \chi}^{\kappa} \cap H_{i} \text { is stationary }\right\}=\theta,
$$

$c$ witnesses $\mathrm{U}(\kappa, \kappa, \theta, \chi)$;

(2) if $\left|\left\{C_{\alpha} \cap \beta \mid \alpha<\kappa\right\}\right|<\kappa$ for all $\beta<\kappa$, then $\mathcal{T}(c)$ is a $\kappa$-tree.

Proof. Let $\operatorname{tr}:[\kappa]^{2} \rightarrow{ }^{<\omega} \kappa$ denote the upper trace function along $\vec{C}$ (recall Definition 4.4). Define a function $h: \kappa \rightarrow \theta$ by letting, for all $\gamma<\kappa$,

$$
h(\gamma):=\sup \left\{i<\theta \mid\left(\operatorname{acc}\left(C_{\gamma}\right) \cup\{\gamma\}\right) \cap H_{i} \neq \emptyset\right\} .
$$

Then, define a coloring $c:[\kappa]^{2} \rightarrow \theta$ by letting, for all $\alpha<\beta<\kappa$,

$$
c(\alpha, \beta):=\max \{h(\tau) \mid \tau \in \operatorname{Im}(\operatorname{tr}(\alpha, \beta))\} .
$$

Claim 4.9.1. $c$ is closed.

Proof. Suppose that $\beta<\kappa, i<\theta$, and $A \subseteq D_{\leq i}^{c}(\beta)$, with $\gamma:=\sup (A)$ smaller than $\beta$. Fix $\alpha \in A$ above $\lambda_{2}(\gamma, \beta)$. By Lemma 4.6, $\operatorname{Im}(\operatorname{tr}(\gamma, \beta)) \subseteq \operatorname{Im}(\operatorname{tr}(\alpha, \beta))$, and hence, by the definition of $c$, we have $c(\gamma, \beta) \leq c(\alpha, \beta) \leq i$, so $\gamma \in D_{\leq i}^{c}(\beta)$.

Claim 4.9.2. Suppose that $\chi \in \operatorname{Reg}(\kappa)$ and

$$
\sup \left\{i<\theta \mid E_{\geq \chi}^{\kappa} \cap H_{i} \text { is stationary }\right\}=\theta .
$$

Then $c$ witnesses $\mathrm{U}(\kappa, \kappa, \theta, \chi)$. Furthermore, for every $i<\theta$, every $\chi^{\prime}<\chi$ and every sequence $\left\langle a_{\gamma} \mid \gamma<\kappa\right\rangle$ with $a_{\gamma} \in[\kappa \backslash \gamma]^{\chi^{\prime}}$ for each $\gamma<\kappa$, there exists a stationary $S \subseteq \kappa$ such that $\min \left(c\left[a_{\gamma} \times a_{\gamma^{\prime}}\right]\right)>i$ for all $\left(\gamma, \gamma^{\prime}\right) \in[S]^{2}$.

Proof. Let $i<\theta$ and $\left\langle a_{\gamma} \mid \gamma<\kappa\right\rangle$ be as above. Find $j>i$ such that $H_{j} \cap E_{\geq \chi}^{\kappa}$ is stationary, and let $\Gamma:=H_{j} \cap E_{\geq \chi}^{\kappa}$. Define $f: \Gamma \rightarrow \kappa$ and $g: \Gamma \rightarrow \kappa$ by letting, for all $\gamma \in \Gamma$,

$$
\begin{aligned}
& \text { - } f(\gamma):=\sup \left\{\lambda_{2}(\gamma, \beta) \mid \beta \in a_{\gamma} \backslash\{\gamma\}\right\} ; \\
& \text { - } g(\gamma):=\sup \left(a_{\gamma}\right) .
\end{aligned}
$$

For each $\gamma \in \Gamma, \operatorname{cf}(\gamma) \geq \chi>\left|a_{\gamma}\right|$, so $f$ is regressive. By Fodor's Lemma, we now pick $\epsilon<\kappa$ for which $S:=\{\gamma \in \Gamma \mid f(\gamma)=\epsilon \& g[\gamma] \subseteq \gamma\}$ is stationary. To see that $S$ is as sought, fix an arbitrary pair $\left(\gamma, \gamma^{\prime}\right) \in[S]^{2}$ and an arbitrary pair $(\alpha, \beta) \in a_{\gamma} \times a_{\gamma^{\prime}}$. There are two cases to consider.

- If $\gamma^{\prime} \in \operatorname{Im}(\operatorname{tr}(\alpha, \beta))$, then since $\gamma^{\prime} \in H_{j}$, we have $c(\alpha, \beta) \geq h\left(\gamma^{\prime}\right) \geq j>i$. 
- Otherwise, we have

$$
\lambda_{2}\left(\gamma^{\prime}, \beta\right) \leq f\left(\gamma^{\prime}\right)=\epsilon<\gamma \leq \alpha<\gamma^{\prime}<\beta,
$$

so by Lemma 4.6, it must be the case that there exists $\delta \in \operatorname{Im}(\operatorname{tr}(\alpha, \beta))$ such that $\gamma^{\prime} \in \operatorname{acc}\left(C_{\delta}\right)$. Altogether, $\gamma^{\prime} \in \operatorname{acc}\left(C_{\delta}\right) \cap H_{j}$, and hence $c(\alpha, \beta) \geq h(\delta) \geq j>i$.

The following claim will now complete the proof of the theorem.

Claim 4.9.3. Suppose that $\left|\left\{C_{\alpha} \cap \beta \mid \alpha<\kappa\right\}\right|<\kappa$ for all $\beta<\kappa$. Then $\mathcal{T}(c)$ is a $\kappa$-tree.

Proof. Suppose not. Pick $\beta<\kappa$ for which $\{c(\cdot, \gamma)\lceil\beta \mid \gamma<\kappa\}$ has size $\kappa$. Pick $\Gamma \in[\kappa]^{\kappa}$ on which the map $\gamma \mapsto c(\cdot, \gamma)\left\lceil\beta\right.$ is one-to-one. Pick a pair $\left(\gamma_{0}, \gamma_{1}\right) \in[\Gamma]^{2}$ for which $\rho_{2}\left(\beta, \gamma_{0}\right)=\rho_{2}\left(\beta, \gamma_{1}\right)$ and the following equalities hold:

- $\left\langle h\left(\operatorname{tr}\left(\beta, \gamma_{0}\right)(n)\right) \mid n<\rho_{2}\left(\beta, \gamma_{0}\right)\right\rangle=\left\langle h\left(\operatorname{tr}\left(\beta, \gamma_{1}\right)(n)\right) \mid n<\rho_{2}\left(\beta, \gamma_{1}\right)\right\rangle$;

- $\left\langle C_{\operatorname{tr}\left(\beta, \gamma_{0}\right)(n)} \cap \beta \mid n \leq \rho_{2}\left(\beta, \gamma_{0}\right)\right\rangle=\left\langle C_{\operatorname{tr}\left(\beta, \gamma_{1}\right)(n)} \cap \beta \mid n \leq \rho_{2}\left(\beta, \gamma_{1}\right)\right\rangle$.

For notational simplicity, write $\left\langle i^{n} \mid n<m\right\rangle$ and $\left\langle C^{n} \mid n \leq m\right\rangle$ for the above common sequences. We shall meet a contradiction by showing that for each $\alpha<\beta$, $c\left(\alpha, \gamma_{0}\right)=c\left(\alpha, \gamma_{1}\right)$. Let $\alpha<\beta$ be arbitrary. The analysis splits into two cases.

- If $C^{n} \cap[\alpha, \beta)=\emptyset$ for all $n \leq m$, then $\min \left(C^{n} \backslash \alpha\right)=\min \left(C^{n} \backslash \beta\right)$ for all $n \leq m$. But then, for each $j<2, \operatorname{Tr}\left(\alpha, \gamma_{j}\right)(n)=\operatorname{Tr}\left(\beta, \gamma_{j}\right)(n)$ for all $n \leq m$. It follows that $\operatorname{Tr}\left(\alpha, \gamma_{j}\right)(m)=\beta, \operatorname{tr}\left(\alpha, \gamma_{j}\right)=\operatorname{tr}\left(\beta, \gamma_{j}\right)^{\frown} \operatorname{tr}(\alpha, \beta)$, and

$$
c\left(\alpha, \gamma_{j}\right)=\max \left\{c\left(\beta, \gamma_{j}\right), c(\alpha, \beta)\right\}=\max \left\{i^{n}, c(\alpha, \beta) \mid n<m\right\} .
$$

As the preceding expression does not depend on $j$, we infer that $c\left(\alpha, \gamma_{0}\right)=c\left(\alpha, \gamma_{1}\right)$.

- Otherwise, let $n \leq m$ be least such that $C^{n} \cap[\alpha, \beta) \neq \emptyset$. Then, as in the previous analysis, for each $j<2$, we have $\operatorname{Tr}\left(\alpha, \gamma_{j}\right)\left\lceil n+1=\operatorname{Tr}\left(\beta, \gamma_{j}\right)\lceil n+1\right.$ and $\operatorname{Tr}\left(\alpha, \gamma_{j}\right)(n+1)=\min \left(C^{n} \backslash \alpha\right)$. Let $\eta:=\min \left(C^{n} \backslash \alpha\right)$. Then, for each $j<2$, we have $\operatorname{tr}\left(\alpha, \gamma_{j}\right)=\operatorname{tr}\left(\eta, \gamma_{j}\right) \frown \operatorname{tr}(\alpha, \eta)$, so

$$
c\left(\alpha, \gamma_{0}\right)=\max \left\{i^{k}, c(\alpha, \eta) \mid k \leq n\right\}=c\left(\alpha, \gamma_{1}\right) .
$$

We can immediately derive a number of corollaries from Theorem 4.9. The first provides, among other things, a complete answer to the question of the existence of closed witnesses to $\mathrm{U}(\ldots)$ at successors of regular cardinals.

Corollary 4.10. Suppose that $\theta, \chi \in \operatorname{Reg}(\kappa)$. Then $(1) \Longrightarrow(2) \Longrightarrow(3) \Longrightarrow$ $(4)$ :

(1) $\kappa$ is the successor of a regular cardinal.

(2) $E_{\geq \chi}^{\kappa}$ admits a non-reflecting stationary set.

(3) There exists a stationary $S \subseteq E_{\geq \chi}^{\kappa}$ such that $\operatorname{Tr}(S) \cap E_{\geq \theta}^{\kappa}$ is nonstationary.

(4) There exists a closed witness to $\mathrm{U}(\kappa, \kappa, \theta, \chi)$.

Proof. (1) $\Longrightarrow(2) \Longrightarrow(3)$ Trivial.

(3) $\Longrightarrow$ (4) Let $S$ be as in (3). Fix a club $D$ in $\kappa$ disjoint from $\operatorname{Tr}(S) \cap E_{\geq \theta}^{\kappa}$ and a $C$-sequence $\vec{C}=\left\langle C_{\alpha} \mid \alpha<\kappa\right\rangle$ such that for all $\alpha \in \operatorname{acc}(\kappa)$,

- $\operatorname{otp}\left(C_{\alpha}\right)=\operatorname{cf}(\alpha)$, and

- if $S \cap D \cap \alpha$ is nonstationary in $\alpha$, then $S \cap D \cap \operatorname{acc}\left(C_{\alpha}\right)=\emptyset$.

Let $\left\langle H_{i} \mid i<\theta\right\rangle$ be an arbitrary partition of $S \cap D$ into stationary sets. Now appeal to Theorem 4.9 . 
Remark 8. If $\kappa$ is the successor of a regular cardinal $\lambda, \square_{\lambda}^{*}$ holds, and $\theta \in \operatorname{Reg}\left(\lambda^{+}\right)$, then we in fact obtain the existence of a closed witness $c$ to $\mathrm{U}(\kappa, \kappa, \theta, \lambda)$ for which $\mathcal{T}(c)$ is a $\kappa$-Aronszajn tree. Likewise, if $\kappa$ is the successor of a singular cardinal $\lambda=2^{<\lambda}, \square_{\lambda}^{*}$ holds, and $E_{\geq \chi}^{\kappa}$ admits a non-reflecting stationary set, then, for each $\theta \in \operatorname{Reg}(\kappa)$, we obtain the existence of a closed witness $c$ to $\mathrm{U}(\kappa, \kappa, \theta, \chi)$ for which $\mathcal{T}(c)$ is a $\kappa$-Aronszajn tree. The proof of these facts comes from a straightforward combination of the proofs of Theorem 4.9, Lemma 2.8 and Corollary 4.10, so we omit it.

It follows that, in the Laver-Shelah model from [LS81] in which $\mathrm{CH}$ (and hence $\square_{\aleph_{1}}^{*}$ ) holds and in which every $\aleph_{2}$-Aronszajn tree is special, for each $n<2$, there is a closed witness $c$ to $\mathrm{U}\left(\aleph_{2}, \aleph_{2}, \aleph_{n}, \aleph_{1}\right)$ for which $\mathcal{T}(c)$ is a special $\aleph_{2}$-Aronszajn tree. Let us show that the other extreme is consistent as well. Recall that a $\lambda^{+}$-tree $\mathcal{T}$ is almost Souslin if, for every antichain $A$ of $\mathcal{T}$, the set of $\alpha \in E_{\operatorname{cf}(\lambda)}^{\lambda^{+}}$such that $A$ has non-empty intersection with the $\alpha^{\text {th }}$ level of $\mathcal{T}$ is nonstationary.

Corollary 4.11. If $\mathrm{V}=\mathrm{L}$, then for every infinite regular cardinal $\lambda$ and every $\theta \in \operatorname{Reg}\left(\lambda^{+}\right)$, there exists a closed witness $c$ to $\mathrm{U}\left(\lambda^{+}, \lambda^{+}, \theta, \lambda\right)$ for which $\mathcal{T}(c)$ is an almost Souslin $\lambda^{+}$-Aronszajn tree.

Proof. Suppose that $\mathrm{V}=\mathrm{L}$ and that $\lambda$ is a infinite regular cardinal. Suppose also that $\lambda$ is uncountable (if $\lambda=\aleph_{0}$, then the proof is similar and slightly easier). By [RS17, Theorem 3.1 and Lemma 2.6], $\bigotimes_{\lambda}^{*}$ holds. Then, by [RS17, Theorem 4.11], there exists a $C$-sequence $\vec{C}=\left\langle C_{\alpha} \mid \alpha<\lambda^{+}\right\rangle$satisfying the following conditions:

- $\operatorname{otp}\left(C_{\alpha}\right) \leq \lambda$ for all $\alpha<\lambda^{+}$;

- for every $\alpha<\lambda^{+}$and $\bar{\alpha} \in \operatorname{acc}\left(C_{\alpha}\right), C_{\bar{\alpha}}=C_{\alpha} \cap \bar{\alpha}$;

- for every stationary $S \subseteq E_{\lambda}^{\lambda^{+}}$, there are $\beta^{\prime} \in S$ and $\beta \in \operatorname{nacc}\left(C_{\beta^{\prime}}\right) \cap S$ such that $C_{\beta^{\prime}} \cap \beta \sqsubseteq C_{\beta}$ (i.e., $C_{\beta}$ is an end-extension of $C_{\beta^{\prime}} \cap \beta$ ).

Let $\kappa:=\lambda^{+}$, and fix $\theta \in \operatorname{Reg}(\kappa)$. Let $\left\langle H_{i} \mid i<\theta\right\rangle$ be some partition of $E_{\lambda}^{\kappa}$ into $\theta$-many stationary sets, and define functions $h$ and $c$ as in the proof of Theorem 4.9. Then $c$ witness $\mathrm{U}(\kappa, \kappa, \theta, \lambda)$, and $\mathcal{T}(c)$ is a $\kappa$-tree. By Proposition 2.7, $\mathcal{T}(c)$ is in fact a $\kappa$-Aronszajn tree.

Finally, to see that $\mathcal{T}(c)$ is almost Souslin, suppose that $\left\langle t_{\beta} \mid \beta \in B\right\rangle$ is a sequence such that $B$ is a stationary subset of $E_{\lambda}^{\lambda^{+}}$and $t_{\beta} \in \mathcal{T}(c) \cap{ }^{\beta} \theta$ for each $\beta \in B$. We need to find $\left(\beta, \beta^{\prime}\right) \in[B]^{2}$ such that $t_{\beta} \subseteq t_{\beta^{\prime}}$. For each $\beta \in B$, pick $\gamma_{\beta} \in[\beta, \kappa)$ such that $t_{\beta}=c\left(\cdot, \gamma_{\beta}\right) \uparrow \beta$. There are two cases (and a few subcases) to consider.

- If $\gamma_{\beta}=\beta$ for stationarily many $\beta \in B$, then let us fix some $i<\theta$ for which the following set is stationary:

$$
S:=\left\{\beta \in B \mid \beta=\gamma_{\beta} \& h(\beta)=i\right\} .
$$

By the choice of $\vec{C}$, we may pick $\beta^{\prime} \in S$ and $\beta \in \operatorname{nacc}\left(C_{\beta^{\prime}}\right) \cap S$ such that $C_{\beta^{\prime}} \cap \beta \sqsubseteq$ $C_{\beta}$. We claim that $t_{\beta} \subseteq t_{\beta^{\prime}}$. To show this, we now fix an arbitrary $\alpha<\beta$ and prove that $c(\alpha, \beta)=c\left(\alpha, \beta^{\prime}\right)$. Let $\tau:=\sup \left(C_{\beta^{\prime}} \cap \beta\right)$, and note that $C_{\beta^{\prime}} \cap(\tau+1)=$ $C_{\beta} \cap(\tau+1)$.

$\gg$ If $\alpha \leq \tau$, then $\operatorname{tr}(\alpha, \beta)=\operatorname{tr}\left(\alpha, \beta^{\prime}\right)$, so $c(\alpha, \beta)=c\left(\alpha, \beta^{\prime}\right)$.

$\longrightarrow$ If $\alpha>\tau$, then $\operatorname{tr}\left(\alpha, \beta^{\prime}\right)=\left\langle\beta^{\prime}\right\rangle^{\frown} \operatorname{tr}(\alpha, \beta)$, and thus $c\left(\alpha, \beta^{\prime}\right)=\max \left\{h\left(\beta^{\prime}\right), c(\alpha, \beta)\right\}$.

But $h\left(\beta^{\prime}\right)=h(\beta) \leq c(\alpha, \beta)$, so $c\left(\alpha, \beta^{\prime}\right)=c(\alpha, \beta)$. 
- If $\gamma_{\beta}>\beta$ for club-many $\beta \in B$, then we may fix $i<\theta, \epsilon<\kappa$ and $t \in$ $\mathcal{T}(c) \cap{ }^{(\epsilon+1)} \theta$ for which the following set is stationary:

$$
S:=\left\{\begin{array}{l|l}
\beta \in B \mid \begin{array}{c}
\beta<\gamma_{\beta} \& c\left(\beta, \gamma_{\beta}\right)=i \& \\
\lambda_{2}\left(\beta, \gamma_{\beta}\right)=\epsilon \& t_{\beta}\lceil(\epsilon+1)=t
\end{array}
\end{array}\right\} .
$$

By the choice of $\vec{C}$, we may pick $\beta^{\prime} \in S$ and $\beta \in \operatorname{nacc}\left(C_{\beta^{\prime}}\right) \cap S$ such that $C_{\beta^{\prime}} \cap \beta \sqsubseteq$ $C_{\beta}$. We claim that $t_{\beta} \subseteq t_{\beta^{\prime}}$. To show this, we now fix an arbitrary $\alpha<\beta$ and prove that $c\left(\alpha, \gamma_{\beta}\right)=c\left(\alpha, \gamma_{\beta^{\prime}}\right)$.

$\rightarrow$ If $\alpha \leq \epsilon$, then $t_{\beta}(\alpha)=t(\alpha)=t_{\beta^{\prime}}(\alpha)$.

$\rightarrow$ If $\alpha>\epsilon$, then, since $\operatorname{cf}(\beta)=\operatorname{cf}\left(\beta^{\prime}\right)=\lambda$, neither $\beta$ nor $\beta^{\prime}$ appears as an accumulation point of $C_{\delta}$ for any $\delta<\kappa$, so, by Lemma 4.6, we have $\operatorname{tr}\left(\alpha, \gamma_{\beta}\right)=$ $\operatorname{tr}\left(\beta, \gamma_{\beta}\right)^{\frown} \operatorname{tr}(\alpha, \beta)$ and $\operatorname{tr}\left(\alpha, \gamma_{\beta^{\prime}}\right)=\operatorname{tr}\left(\beta^{\prime}, \gamma_{\beta^{\prime}}\right) \frown \operatorname{tr}\left(\alpha, \beta^{\prime}\right)$. Consequently, $c\left(\alpha, \gamma_{\beta}\right)=$ $\max \left\{c\left(\beta, \gamma_{\beta}\right), c(\alpha, \beta)\right\}$ and $c\left(\alpha, \gamma_{\beta^{\prime}}\right)=\max \left\{c\left(\beta^{\prime}, \gamma_{\beta^{\prime}}\right), c\left(\alpha, \beta^{\prime}\right)\right\}$. As $\beta \in \operatorname{nacc}\left(C_{\beta^{\prime}}\right)$ and $C_{\beta}^{\prime} \cap \beta \sqsubseteq C_{\beta}$, the previous analysis shows that $c(\alpha, \beta)=c\left(\alpha, \beta^{\prime}\right)$. By our choice of $S$, we also have $c\left(\beta, \gamma_{\beta}\right)=i=c\left(\beta^{\prime}, \gamma_{\beta^{\prime}}\right)$. Putting this together, we obtain $c\left(\alpha, \gamma_{\beta}\right)=c\left(\alpha, \gamma_{\beta^{\prime}}\right)$.

Corollary 4.12. Suppose that $\sigma, \theta \in \operatorname{Reg}(\kappa)$. If $\square\left(\kappa, \sqsubseteq_{\sigma}\right)$ holds, then there exists a closed witness $c:[\kappa]^{2} \rightarrow \theta$ to $\mathrm{U}(\kappa, \kappa, \theta, \sup (\operatorname{Reg}(\kappa)))$. Furthermore, if $\sigma=\aleph_{0}$ (i.e., if $\square(\kappa)$ holds), or if $\kappa$ is $<\sigma$-inaccessible, then $\mathcal{T}(c)$ is a $\kappa$-Aronszajn tree.

Proof. By [BR19, Theorem 1.24], $\square\left(\kappa, \sqsubseteq_{\sigma}\right)$ entails a $C$-sequence $\left\langle C_{\alpha} \mid \alpha<\kappa\right\rangle$ and a partition $\left\langle F_{i} \mid i<\kappa\right\rangle$ of $\kappa$ into fat sets such that, for every $i<\kappa$, every $\alpha \in F_{i} \cap E_{\geq \sigma}^{\kappa}$, and every $\bar{\alpha} \in \operatorname{acc}\left(C_{\alpha}\right)$, we have $\bar{\alpha} \in F_{i}$ and $C_{\bar{\alpha}}=C_{\alpha} \cap \bar{\alpha}$. For all $\alpha \in E_{\geq \sigma}^{\kappa}$, let $c_{\alpha}:=C_{\alpha}$. For all $\alpha \in E_{<\sigma}^{\kappa}$, let $c_{\alpha}$ be some club in $\alpha$ of order type less than $\sigma$. For all $i<\theta$, let $H_{i}:=F_{i} \cap E_{\geq \sigma}^{\kappa}$. Now appeal to Theorem 4.9 with $\vec{C}=\left\langle c_{\alpha} \mid \alpha<\kappa\right\rangle$ and $\left\langle H_{i} \mid i<\theta\right\rangle$ to obtain the desired coloring, $c:[\kappa]^{2} \rightarrow \theta$.

Finally, if $\sigma=\aleph_{0}$, or if $\kappa$ is $<\sigma$-inaccessible, then the hypothesis of Clause (2) of Lemma 4.9 holds. So, by Proposition 2.7, in these cases, $\mathcal{T}(c)$ is a $\kappa$-Aronszajn tree.

Remark 9. By [LHL19], $\square(\kappa)$ entails the principle $\square^{\text {ind }}(\kappa, \theta)$ for every $\theta \in \operatorname{Reg}(\kappa)$. In Part III, we shall see that $\square^{\text {ind }}(\kappa, \theta)$ yields the existence of a closed witness to $\mathrm{U}(\kappa, \kappa, \theta, \sup (\operatorname{Reg}(\kappa)))$ which is, moreover, subadditive.

Since Corollary 4.10 fully answers the question about the existence of closed witnesses to $\mathrm{U}(\ldots)$ at successors of regular cardinals, we spend the remainder of this section investigating, in turn, successors of singular cardinals and inaccessible cardinals.

4.4. Successors of singular cardinals. We begin this subsection with an immediate corollary to Theorem 4.9 that can be seen as a counterpart to Corollary 4.10.

Corollary 4.13. Suppose that $\lambda$ is a singular cardinal. Then there exists a closed witness to $\mathrm{U}\left(\lambda^{+}, \lambda^{+}, \operatorname{cf}(\lambda), \lambda\right)$. Furthermore, if $\square_{\lambda}^{*}$ holds, then there exists a closed witness c to $\mathrm{U}\left(\lambda^{+}, \lambda^{+}, \operatorname{cf}(\lambda), \lambda\right)$, for which $\mathcal{T}(c)$ is a $\lambda^{+}$-Aronszajn tree.

Proof. Set $\kappa:=\lambda^{+}$and $\theta:=\operatorname{cf}(\lambda)$. Fix a $C$-sequence $\vec{C}=\left\langle C_{\alpha} \mid \alpha<\kappa\right\rangle$ with $\operatorname{otp}\left(C_{\alpha}\right)<\lambda$ for all $\alpha<\kappa$. If $\square_{\lambda}^{*}$ holds, then we moreover require that $\mid\left\{C_{\alpha} \cap \beta \mid\right.$ $\alpha<\kappa\} \mid \leq \lambda$ for all $\beta<\kappa$. Together with Proposition 2.7, this will ensure that, under $\square_{\lambda}^{*}$, the associated tree $\mathcal{T}(c)$ will be $\lambda^{+}$-Aronszajn. 
Let $\left\langle\lambda_{i} \mid i<\theta\right\rangle$ be a strictly increasing sequence of infinite regular cardinals that converges to $\lambda$. For all $i<\theta$, let $H_{i}:=E_{\lambda_{i}}^{\kappa}$. It is clear that, for every $\chi \in \operatorname{Reg}(\lambda)$, we have $\sup \left\{i<\theta \mid E_{\geq \chi}^{\kappa} \cap H_{i}\right.$ is stationary $\}=\theta$. Moreover, for each $\alpha \in \operatorname{acc}(\kappa)$, there is $j<\theta$ such that $\operatorname{otp}\left(C_{\alpha}\right)<\lambda_{j}$, and hence $\sup \left\{i<\theta \mid \operatorname{acc}\left(C_{\alpha}\right) \cap H_{i} \neq \emptyset\right\} \leq j<\theta$, so we may appeal to Theorem 4.9 to obtain a coloring $c$ as desired.

Remark 10. If $\lambda$ is a singular cardinal, $\square_{\lambda}$ holds and $2^{\lambda}=\lambda^{+}$, then for every $\theta \in \operatorname{Reg}(\lambda)$, there exists a closed witness $c$ to $\mathrm{U}\left(\lambda^{+}, \lambda^{+}, \theta, \lambda\right)$ for which $\mathcal{T}(c)$ is a nonspecial $\lambda^{+}$-Aronszajn tree. The proof follows the arguments of the proofs of Corollaries 4.12 and 4.11, building on Theorems 5.1, 5.3 and 1.24 of [BR19].

Note that Theorem 2.11 provides some limits on the extent of positive ZFC results regarding the existence of closed witnesses to $U(. .$.$) at successors of singular$ cardinals. The rest of this subsection is devoted to obtaining positive results under additional assumptions about the cardinals under consideration. Note first that, by Corollary 4.10, if $\lambda$ is a singular cardinal, $\theta, \chi \in \operatorname{Reg}(\lambda)$, and there is a stationary $S \subseteq E_{\geq \chi}^{\lambda^{+}}$such that $\operatorname{Tr}(S) \cap E_{\geq \theta}^{\lambda^{+}}$is nonstationary, then there is a closed witness to $\mathrm{U}\left(\lambda^{+}, \lambda^{+}, \theta, \chi\right)$. The following theorem provides an improvement to this observation by weakening the hypotheses.

Theorem 4.14. Suppose that $\lambda$ is a singular cardinal, $\theta, \chi \in \operatorname{Reg}(\lambda)$, and there exists a stationary $S \subseteq E_{\geq \chi}^{\lambda^{+}}$such that

$$
\sup \left\{\nu<\lambda \mid \operatorname{Tr}(S) \cap E_{\nu}^{\lambda^{+}} \text {is stationary }\right\}<\lambda .
$$

Then there exists a closed witness to $\mathrm{U}\left(\lambda^{+}, \lambda^{+}, \theta, \chi\right)$.

Proof. By Corollary 4.10, we may assume that every stationary subset of $E_{\geq \chi}^{\lambda^{+}}$ reflects. We start by showing that we can find a stationary set as in the statement of the theorem that is slightly better-behaved. In particular, we find a stationary set that concentrates on a cofinality different from both $\aleph_{0}$ and $\operatorname{cf}(\lambda)$.

Claim 4.14.1. There exist $\sigma \in \operatorname{Reg}(\lambda) \backslash \chi$ with $\sigma \notin\left\{\aleph_{0}, \operatorname{cf}(\lambda)\right\}$ and a stationary $\Delta \subseteq E_{\sigma}^{\lambda^{+}}$, for which $\sup \left\{\operatorname{cf}(\tau) \mid \tau<\lambda^{+}, \Delta \cap \tau\right.$ is stationary $\}<\lambda$.

Proof. By the hypothesis of the theorem, we may fix a stationary $S_{0} \subseteq E_{\geq \chi}^{\lambda^{+}}$and a club $D \subseteq \lambda^{+}$such that

$$
\sup \left\{\operatorname{cf}(\tau) \mid \tau \in D \& S_{0} \cap \tau \text { is stationary }\right\}<\lambda .
$$

Using Fodor's Lemma, and by shrinking $S_{0}$ if necessary, we may assume that $S_{0} \subseteq D$ and there is $\sigma_{0} \in \operatorname{Reg}(\lambda)$ such that $S_{0} \subseteq E_{\sigma_{0}}^{\lambda^{+}}$. It is clear that $\sigma_{0} \geq \chi$, so, by assumption, $\operatorname{Tr}\left(S_{0}\right)$ is stationary. By another application of Fodor's Lemma, we may find a stationary set $S_{1}$ for which there is $\sigma_{1} \in \operatorname{Reg}(\lambda)$ such that $S_{1} \subseteq \operatorname{Tr}\left(S_{0}\right) \cap E_{\sigma_{1}}^{\lambda^{+}}$. Clearly, $\operatorname{Tr}\left(S_{1}\right) \subseteq \operatorname{Tr}\left(S_{0}\right)$ and $\sigma_{1}>\sigma_{0}$. Doing this again, find a stationary set $S_{2}$ for which there is $\sigma_{2} \in \operatorname{Reg}(\lambda)$ such that $S_{2} \subseteq \operatorname{Tr}\left(S_{1}\right) \cap E_{\sigma_{2}}^{\lambda^{+}}$. Altogether $\operatorname{Tr}\left(S_{2}\right) \subseteq \operatorname{Tr}\left(S_{1}\right) \subseteq \operatorname{Tr}\left(S_{0}\right) \subseteq D$ and $\sigma_{2}>\sigma_{1}>\sigma_{0}$. There must be $i<3$ for which $\sigma_{i} \notin\left\{\aleph_{0}, \operatorname{cf}(\lambda)\right\}$. Choose such an $i$, and note that $\sigma:=\sigma_{i}$ and $\Delta:=S_{i}$ are as sought.

Let $\sigma$ and $\Delta$ be given by the preceding claim, and fix $\mu \in \operatorname{Reg}(\lambda)$ such that

$$
\max \left\{\sigma, \sup \left\{\operatorname{cf}(\tau) \mid \tau<\lambda^{+} \& \Delta \cap \tau \text { is stationary }\right\}\right\}<\mu .
$$


Fix a function $h: \lambda^{+} \rightarrow \theta$ such that, for all $i<\theta, H_{i}:=\left\{\gamma \in E_{\mu}^{\lambda^{+}} \mid h(\gamma)=i\right\}$ is stationary.

Claim 4.14.2. There exists a $C$-sequence $\left\langle e_{\delta} \mid \delta<\lambda^{+}\right\rangle$such that

- for all $\delta \in \operatorname{acc}\left(\lambda^{+}\right)$, otp $\left(e_{\delta}\right)=\operatorname{cf}(\delta)$;

- for all $\delta \in E_{\geq \mu}^{\lambda^{+}}$, we have $e_{\delta} \cap \Delta=\emptyset$;

- for every club $D \subseteq \lambda^{+}$and every $i<\theta$, there exists $\delta \in \Delta$ such that $\sup \left(e_{\delta} \cap H_{i} \cap D\right)=\delta$.

Proof. It is clear how to obtain $e_{\delta}$ for each $\delta \in \lambda^{+} \backslash \Delta$. Now, to deal with $\delta \in \Delta$, proceed as follows. Let $\left\langle S_{i} \mid i<\theta\right\rangle$ be some partition of $\Delta$ into stationary sets. For each $i<\theta$, since $S_{i}$ is a stationary subset of $E_{\neq \operatorname{cf}(\lambda)}^{\lambda^{+}}$, [Rin10, Proposition 1.4] implies that $\mathbf{s}^{-}\left(S_{i}\right)$ holds. Thus, as pointed out on top of page 145 of [Rin11], there exists a sequence $\left\langle B_{\delta} \mid \delta \in S_{i}\right\rangle$, with $\sup \left(B_{\delta}\right)=\delta$ for each $\delta \in S_{i}$, such that for every club $D \subseteq \lambda^{+}$, the set $\left\{\delta \in S_{i} \mid B_{\delta} \subseteq D \cap H_{i}\right\}$ is stationary. Now, for each $i<\theta$ and $\delta \in S_{i}$, pick a club $e_{\delta}$ in $\delta$ of order-type $\sigma$ with nacc $\left(e_{\delta}\right) \subseteq B_{\delta}$.

For each $\alpha<\lambda^{+}$, define a sequence $\left\langle C_{\alpha}^{n} \mid n<\omega\right\rangle$ by recursion on $n<\omega$ as follows:

- $C_{\alpha}^{0}:=e_{\alpha}$

- $C_{\alpha}^{n+1}:=\operatorname{cl}\left(C_{\alpha}^{n} \cup \bigcup\left\{e_{\delta} \mid \delta \in \operatorname{acc}\left(C_{\alpha}^{n}\right) \cap \Delta\right\}\right)$.

Then, let $C_{\alpha}:=\operatorname{cl}\left(\bigcup_{n<\omega} C_{\alpha}^{n}\right)$.

Claim 4.14.3. All of the following hold.

(1) $\vec{C}:=\left\langle C_{\alpha} \mid \alpha<\lambda^{+}\right\rangle$is a $C$-sequence.

(2) For all $\alpha \in \operatorname{acc}\left(\lambda^{+}\right)$, otp $\left(C_{\alpha}\right)=\operatorname{cf}(\alpha)$.

(3) For all $\alpha \in \operatorname{acc}\left(\lambda^{+}\right)$and $\delta \in\left(\operatorname{acc}\left(C_{\alpha}\right) \cup\{\alpha\}\right) \cap \Delta$, we have $e_{\delta} \subseteq C_{\alpha}$.

(4) For all $\alpha \in E_{\geq \mu}^{\lambda^{+}}$, we have $C_{\alpha} \cap \Delta=\emptyset$.

Proof. It is easy to see that $C_{\alpha}=e_{\alpha}$ for all $\alpha \in E_{\leq \sigma}^{\lambda^{+}} \cup E_{\geq \mu}^{\lambda^{+}}$. Now, for all $\alpha<\lambda^{+}$ with $\sigma<\operatorname{cf}(\alpha)<\mu$, we have $\operatorname{otp}\left(C_{\alpha}^{n}\right)=\operatorname{cf}(\alpha) \cdot \sigma=\operatorname{cf}(\alpha)$ for all $n<\omega$.

We now perform walks along $\vec{C}$ and derive a closed coloring $c:\left[\lambda^{+}\right]^{2} \rightarrow \theta$ as in the proof of Theorem 4.9 by letting, for all $\alpha<\beta<\lambda^{+}$,

$$
c(\alpha, \beta):=\max \{h(\tau) \mid \tau \in \operatorname{Im}(\operatorname{tr}(\alpha, \beta))\} .
$$

By the implication $(2) \Longrightarrow(3)$ of Lemma 4.2 , the following claim suffices to finish the proof of the theorem.

Claim 4.14.4. Suppose that $\mathcal{A} \subseteq\left[\lambda^{+}\right]^{<\chi}$ is a family consisting of $\lambda^{+}$-many pairwise disjoint sets, $D$ is a club in $\lambda^{+}$, and $i<\theta$. Then there exist $\gamma \in D, a \in \mathcal{A}$, and $\epsilon<\gamma$ such that

- $\gamma<a$;

- for all $\alpha \in(\epsilon, \gamma)$ and all $\beta \in a$, we have $c(\alpha, \beta)>i$.

Proof. Fix $\delta \in \Delta$ such that $\sup \left(e_{\delta} \cap H_{i+1} \cap D\right)=\delta$, and then fix an arbitrary $a \in \mathcal{A}$ with $\delta<a$. Let $\Lambda:=\sup \left\{\lambda_{2}(\delta, \beta) \mid \beta \in a\right\}$. As $\operatorname{cf}(\delta)=\sigma \geq \chi>|a|$, we have $\Lambda<\delta$, so we may pick $\gamma \in e_{\delta} \cap H_{i+1} \cap D$ above $\Lambda$.

Let $T:=\{\delta\} \cup \bigcup_{\beta \in a} \operatorname{Im}(\operatorname{tr}(\delta, \beta))$, and let

$$
\epsilon:=\sup \left\{\Lambda, \sup \left(C_{\tau} \cap \gamma\right) \mid \tau \in T \& \sup \left(C_{\tau} \cap \gamma\right)<\gamma\right\} .
$$


Since $\operatorname{cf}(\gamma)=\mu>\chi>|a|$, we have $\epsilon<\gamma$. We claim that $\gamma, a$, and $\epsilon$ are as desired. To verify this, let $\alpha \in(\epsilon, \gamma)$ and $\beta \in a$ be arbitrary. We will show that $\gamma \in \operatorname{tr}(\alpha, \beta)$, and hence $c(\alpha, \beta) \geq h(\gamma)>i$. Let $\ell:=\rho_{2}(\delta, \beta)$. By Lemma 4.6, we have $\operatorname{tr}(\delta, \beta) \sqsubseteq \operatorname{tr}(\alpha, \beta)$, and there are two cases to consider.

- If $\delta \in \operatorname{nacc}\left(C_{\operatorname{tr}(\delta, \beta)(\ell-1)}\right)$, then, since $\operatorname{tr}(\alpha, \beta)(\ell-1)=\operatorname{tr}(\delta, \beta)(\ell-1)$ and

$$
\sup \left(C_{\operatorname{tr}(\delta, \beta)(\ell-1)} \cap \delta\right) \leq \lambda_{2}(\delta, \beta)<\alpha<\gamma<\delta,
$$

we have $\operatorname{tr}(\alpha, \beta)(\ell)=\min \left(C_{\operatorname{tr}(\delta, \beta)(\ell-1)} \backslash \alpha\right)=\delta$. As otp $\left(C_{\delta}\right)=\operatorname{cf}(\delta)=\sigma<\mu=$ $\operatorname{cf}(\gamma)$, we have $\sup \left(C_{\delta} \cap \gamma\right)<\gamma$. As $\delta \in T$, we then have $\sup \left(C_{\delta} \cap \gamma\right) \leq \epsilon<\alpha<\gamma$. Finally, since $\gamma \in e_{\delta} \subseteq C_{\delta}$, we have $\operatorname{tr}(\alpha, \beta)(\ell+1)=\min \left(C_{\delta} \backslash \alpha\right)=\gamma$.

- If $\delta \in \operatorname{acc}\left(C_{\operatorname{tr}(\delta, \beta)(\ell-1)}\right)$, then, by Claim 4.14.3(4), it follows that $\operatorname{cf}(\operatorname{tr}(\delta, \beta)(\ell-$ 1) $)<\mu=\operatorname{cf}(\gamma)$, and hence $\sup \left(C_{\operatorname{tr}(\delta, \beta)(\ell-1)} \cap \gamma\right) \leq \epsilon<\alpha<\gamma$. Consequently, $\operatorname{tr}(\alpha, \beta)(\ell)=\min \left(C_{\operatorname{tr}(\delta, \beta)(\ell-1)} \backslash \alpha\right)=\gamma$.

We next show that the existence of closed witnesses to $\mathrm{U}\left(\lambda^{+}, \lambda^{+}, \theta, \operatorname{cf}(\lambda)\right)$ follows from a local instance of $\mathrm{GCH}$.

Theorem 4.15. Suppose that $\lambda$ is a singular cardinal, $\theta \in \operatorname{Reg}(\lambda)$, and $2^{\lambda}=\lambda^{+}$. Then there exists a closed witness to $\mathrm{U}\left(\lambda^{+}, \lambda^{+}, \theta, \operatorname{cf}(\lambda)\right)$.

Proof. Let $\chi:=\max \{\theta, \operatorname{cf}(\lambda)\}^{+}$and $\Delta:=E_{\chi}^{\lambda^{+}}$. As $\Delta$ is a stationary subset of $E_{\neq \mathrm{cf}(\lambda)}^{\lambda^{+}}$and $2^{\lambda}=\lambda^{+}$, [She10, Claim 2.3] provides us with a $\diamond(\Delta)$-sequence, $\left\langle X_{\delta}\right|$ $\delta \in \Delta\rangle$.

Let $\left\langle\lambda_{j} \mid j<\operatorname{cf}(\lambda)\right\rangle$ be a strictly increasing sequence of regular cardinals converging to $\lambda$, with $\lambda_{0}>\chi$. Fix a function $h: \lambda^{+} \rightarrow \theta$ such that for every $i<\theta$ and $j<\operatorname{cf}(\lambda)$, the following set is stationary:

$$
H_{j}^{i}:=\left\{\gamma \in E_{\lambda_{j}}^{\lambda^{+}} \mid h(\gamma)=i\right\} .
$$

Now, let $\left\langle e_{\delta} \mid \delta<\lambda^{+}\right\rangle$be a $C$-sequence such that

- for all $\delta \in \operatorname{acc}\left(\lambda^{+}\right), \operatorname{otp}\left(e_{\delta}\right)=\operatorname{cf}(\delta)$;

- for all $\delta \in \Delta, i<\theta$, and $j<\operatorname{cf}(\lambda)$, if $\sup \left(X_{\delta} \cap H_{j}^{i}\right)=\delta$, then $\sup \left(X_{\delta} \cap\right.$ $\left.H_{j}^{i} \cap e_{\delta}\right)=\delta$.

For each $\alpha<\lambda^{+}$, define a sequence $\left\langle C_{\alpha}^{n} \mid n<\omega\right\rangle$ by recursion on $n<\omega$ as follows:

- $C_{\alpha}^{0}:=e_{\alpha}$

- $C_{\alpha}^{n+1}:=\operatorname{cl}\left(C_{\alpha}^{n} \cup \bigcup\left\{e_{\delta} \mid \delta \in \operatorname{acc}\left(C_{\alpha}^{n}\right) \cap \Delta\right\}\right)$.

Then, let $C_{\alpha}:=\operatorname{cl}\left(\bigcup_{n<\omega} C_{\alpha}^{n}\right)$.

Claim 4.15.1. All of the following hold.

(1) $\vec{C}:=\left\langle C_{\alpha} \mid \alpha<\lambda^{+}\right\rangle$is a $C$-sequence.

(2) For all $\alpha \in \operatorname{acc}\left(\lambda^{+}\right), \operatorname{otp}\left(C_{\alpha}\right)<\lambda$.

(3) For all $\alpha \in \operatorname{acc}\left(\lambda^{+}\right)$and $\delta \in\left(\operatorname{acc}\left(C_{\alpha}\right) \cup\{\alpha\}\right) \cap \Delta$, $e_{\delta} \subseteq C_{\alpha}$.

(4) For every club $D$ in $\lambda^{+}$, there is $\delta \in \Delta$ such that for every $\mu<\lambda, \Lambda<\delta$, and $i<\theta$, there is $\gamma \in e_{\delta} \cap D$ such that $\operatorname{cf}(\gamma)>\mu, \gamma>\Lambda$ and $h(\gamma)=i$.

Proof. Clause (1) is straightforward.

(2) Fix $\alpha \in \operatorname{acc}\left(\lambda^{+}\right) \cdot\left|C_{\alpha}^{0}\right|=\operatorname{cf}(\alpha)$ and, by induction on $n$, it is then easy to see that, for all $n<\omega$, we have $\left|C_{\alpha}^{n+1}\right| \leq \operatorname{cf}(\alpha) \cdot \chi$. It follows that $\left|C_{\alpha}\right| \leq \operatorname{cf}(\alpha) \cdot \chi<\lambda$. 
(3) For each $\alpha \in \Delta$, since otp $\left(e_{\alpha}\right)=\operatorname{cf}(\alpha)=\chi$, we simply have $\operatorname{acc}\left(C_{\alpha}^{n}\right) \cap \Delta=\emptyset$ for all $n<\omega$, so $C_{\alpha}=e_{\alpha}$. Now, for $\alpha \in \operatorname{acc}\left(\lambda^{+}\right)$and $\delta \in \operatorname{acc}\left(C_{\alpha}\right)$, since $\operatorname{cf}(\delta)=\chi>$ $\omega$, there must exist some $n<\omega$ such that $\delta \in \operatorname{acc}\left(C_{\alpha}^{n}\right)$, and hence $e_{\delta} \subseteq C_{\alpha}^{n+1} \subseteq C_{\alpha}$.

(4) Fix a club $D$ in $\lambda^{+}$. For each $i<\theta$ and $j<\operatorname{cf}(\lambda), H_{j}^{i}$ is stationary, so

$$
E:=\bigcap_{i<\theta} \bigcap_{j<\operatorname{cf}(\lambda)} \operatorname{acc}^{+}\left(D \cap H_{j}^{i}\right)
$$

is a club. Since $\left\{\delta \in \Delta \mid D \cap \delta=X_{\delta}\right\}$ is stationary, we can pick $\delta \in \Delta \cap E$ such that $D \cap \delta=X_{\delta}$. For all $i<\theta$ and $j<\operatorname{cf}(\lambda)$, we have $\sup \left(X_{\delta} \cap H_{j}^{i}\right)=\delta$, and hence $\sup \left(X_{\delta} \cap H_{j}^{i} \cap e_{\delta}\right)=\delta$. In particular, for every $\mu<\lambda, \Lambda<\delta$, and $i<\theta$, we may fix some $j<\operatorname{cf}(\lambda)$ such that $\lambda_{j}>\mu$ and then find $\gamma \in X_{\delta} \cap H_{j}^{i} \cap e_{\delta}$ above $\Lambda$. Clearly, $\operatorname{cf}(\gamma)>\mu, h(\gamma)=i$, and $\gamma$ is an accumulation point of the club $D$

We now perform walks along $\vec{C}$ and derive a closed coloring $c:[\kappa]^{2} \rightarrow \theta$ as in the proof of Theorem 4.9 by letting, for all $\alpha<\beta<\lambda^{+}$,

$$
c(\alpha, \beta):=\max \{h(\tau) \mid \tau \in \operatorname{Im}(\operatorname{tr}(\alpha, \beta))\} .
$$

We claim that $c$ witnesses $\mathrm{U}\left(\lambda^{+}, \lambda^{+}, \theta, \operatorname{cf}(\lambda)\right)$ and prove this by verifying Clause (2) of Lemma 4.2. To this end, fix a family $\mathcal{A} \subseteq\left[\lambda^{+}\right]^{<\operatorname{cf}(\lambda)}$ consisting of $\lambda^{+}$-many pairwise disjoint sets, a club $D$ in $\lambda^{+}$, and a color $i<\theta$. We will find $\gamma \in D, a \in \mathcal{A}$, and $\epsilon<\gamma$ such that

- $\gamma<a$;

- for all $\alpha \in(\epsilon, \gamma)$ and all $\beta \in a$, we have $c(\alpha, \beta)>i$.

Use Clause (4) of Claim 4.15.1 to find $\delta \in \Delta$ such that, for every $\mu<\lambda$ and $\Lambda<\delta$, there exists $\gamma \in e_{\delta} \cap D$ such that $\operatorname{cf}(\gamma)>\mu, \gamma>\Lambda$, and $h(\gamma)=i+1$. Fix an arbitrary $a \in \mathcal{A}$ with $\delta<a$, and set

- $\Lambda:=\sup \left\{\lambda_{2}(\delta, \beta) \mid \beta \in a\right\}$

- $C:=C_{\delta} \cup \bigcup_{\beta \in a} \bigcup_{\tau \in \operatorname{Im}(\operatorname{tr}(\delta, \beta))} C_{\tau}$.

As $|a|<\operatorname{cf}(\lambda)<\operatorname{cf}(\delta)$, we have $\Lambda<\delta$ and $|C|<\lambda$. Thus, we can pick $\gamma \in e_{\delta} \cap D$ such that $\operatorname{cf}(\gamma)>|C|, \gamma>\Lambda$, and $h(\gamma)=i+1$. Let $\epsilon:=\max \{\Lambda, \sup (C \cap \gamma)\}$.

We claim that $\gamma, a$, and $\epsilon$ are as desired. To this end, let $\alpha \in(\epsilon, \gamma)$ and $\beta \in a$ be arbitrary. Then

$$
\lambda_{2}(\delta, \beta) \leq \Lambda \leq \epsilon<\alpha<\gamma<\delta<\beta,
$$

so, by Lemma 4.6, $\operatorname{tr}(\delta, \beta) \sqsubseteq \operatorname{tr}(\alpha, \beta)$. We claim that $\gamma \in \operatorname{Im}(\operatorname{tr}(\alpha, \beta))$. To see this, let $\ell:=\rho_{2}(\delta, \beta)$, and consider the following two cases, each of which will involve the use of Clause (3) of Claim 4.15.1.

- If $\delta \in \operatorname{nacc}\left(C_{\operatorname{tr}(\delta, \beta)(\ell-1)}\right)$, then $\sup \left(C_{\operatorname{tr}(\delta, \beta)(\ell-1)} \cap \delta\right) \leq \lambda_{2}(\delta, \beta)$, and hence

$$
[\alpha, \delta) \cap C_{\operatorname{tr}(\delta, \beta)(\ell-1)} \subseteq\left(\lambda_{2}(\delta, \beta), \delta\right) \cap C_{\operatorname{tr}(\delta, \beta)(\ell-1)}=\emptyset .
$$

Consequently, $\operatorname{tr}(\alpha, \beta)(\ell)=\min \left(C_{\operatorname{tr}(\alpha, \beta)(\ell-1)} \backslash \alpha\right)=\min \left(C_{\operatorname{tr}(\delta, \beta)(\ell-1)} \backslash \alpha\right)=\delta$. As

$$
[\alpha, \gamma) \cap C_{\operatorname{tr}(\alpha, \beta)(\ell) \subseteq(\epsilon, \gamma) \cap C_{\delta}=\emptyset}
$$

and $\gamma \in e_{\delta} \subseteq C_{\delta} \subseteq C$, we have $\operatorname{tr}(\alpha, \beta)(\ell+1)=\min \left(C_{\delta} \backslash \alpha\right)=\gamma$.

- If $\delta \in \operatorname{acc}\left(C_{\operatorname{tr}(\delta, \beta)(\ell-1)}\right)$, then

$$
[\alpha, \gamma) \cap C_{\operatorname{tr}(\delta, \beta)(\ell-1)} \subseteq(\epsilon, \gamma) \cap C=\emptyset .
$$

As $\delta \in \operatorname{acc}\left(C_{\operatorname{tr}(\delta, \beta)(\ell-1)}\right) \cap \Delta$, we have $\gamma \in e_{\delta} \subseteq C_{\operatorname{tr}(\delta, \beta)(\ell-1)}$, and hence $\operatorname{tr}(\alpha, \beta)(\ell)=$ $\min \left(C_{\operatorname{tr}(\delta, \beta)(\ell-1) \backslash \alpha)=\gamma}\right.$. 
In either case, we have shown that $\gamma \in \operatorname{Im}(\operatorname{tr}(\alpha, \beta))$, and hence $c(\alpha, \beta) \geq h(\gamma)=$ $i+1$.

Our final result of this subsection shows that a failure of the simultaneous stationary reflection principle $\operatorname{Refl}\left(<\operatorname{cf}(\lambda), \lambda^{+}\right)$entails the existence of a closed witness to $\mathrm{U}\left(\lambda^{+}, \lambda^{+}, \theta, \operatorname{cf}(\lambda)\right)$ for all $\theta \in \operatorname{Reg}(\lambda)$.

Theorem 4.16. Suppose that $\lambda$ is a singular cardinal and $\theta \in \operatorname{Reg}(\lambda)$. If any one of the following conditions holds:

(1) $\operatorname{Refl}\left(<\operatorname{cf}(\lambda), \lambda^{+}\right)$fails;

(2) $\operatorname{cf}\left(\mathrm{NS}_{\mathrm{cf}(\lambda)}, \subseteq\right)<\lambda$ and $\theta<\operatorname{cf}(\lambda)$;

(3) there exists a tail-closed witness to $\mathrm{U}\left(\lambda^{+}, 2, \theta, 2\right)$;

(4) there exists a somewhere-closed witness to $\mathrm{U}\left(\lambda^{+}, 2, \theta, \omega\right)$;

then there exists a closed witness to $\mathrm{U}\left(\lambda^{+}, \lambda^{+}, \theta, \operatorname{cf}(\lambda)\right)$.

The rest of this subsection is dedicated to the proof of Theorem 4.16. The proof splits into two cases based on whether $\lambda$ has uncountable or countable cofinality. The structures of the proofs in the two cases are similar to one another. We begin by identifying a useful club-guessing sequence (or, in the countable cofinality case, an "off-center" club-guessing matrix) and its associated ideal. We use these objects to identify a $C$-sequence (or, in the countable cofinality case, a collection of $C$ sequences) along which we will perform walks. After isolating the salient properties of walks along these $C$-sequences, we will verify, in turn, that each of the conditions identified in the statement of the theorem implies the existence of a closed witness to $\mathrm{U}\left(\lambda^{+}, \lambda^{+}, \theta, \operatorname{cf}(\lambda)\right)$. Let us begin.

Case 1: Uncountable cofinality. Assume in this case that $\operatorname{cf}(\lambda)>\omega$. By [ES09, Theorem 2], we may find a stationary $\Delta \subseteq E_{\mathrm{cf}(\lambda)}^{\lambda^{+}}$and a sequence $\vec{e}=\left\langle e_{\delta}\right|$ $\delta \in \Delta\rangle$ such that

- for every $\delta \in \Delta, e_{\delta}$ is a club in $\delta$ of order type $\operatorname{cf}(\lambda)$;

- for every $\delta \in \Delta,\left\langle\operatorname{cf}(\gamma) \mid \gamma \in \operatorname{nacc}\left(e_{\delta}\right)\right\rangle$ is strictly increasing and converging to $\lambda$;

- for every club $D$ in $\lambda^{+}$, there exists $\delta \in \Delta$ such that $e_{\delta} \subseteq D$.

Now, define $\mathcal{I} \subseteq \mathcal{P}\left(\lambda^{+}\right)$by letting $A \in \mathcal{P}\left(\lambda^{+}\right)$be in $\mathcal{I}$ iff there exists a club $D \subseteq \lambda^{+}$such that for every $\delta \in \Delta \cap D$, we have $\sup \left(\operatorname{nacc}\left(e_{\delta}\right) \cap D \cap A\right)<\delta$.

Claim 4.16.1. I satisfies all of the following properties:

(a) $\mathcal{I}$ is a $\operatorname{cf}(\lambda)$-complete proper ideal over $\lambda^{+}$, extending $\mathrm{NS}_{\lambda^{+}}$;

(b) $\mathcal{I}$ is $\tau$-indecomposable for all $\tau \in \operatorname{Reg}(\lambda) \backslash\{\operatorname{cf}(\lambda)\}$;

(c) if $\operatorname{cf}\left(\mathrm{NS}_{\operatorname{cf}(\lambda)}, \subseteq\right)<\lambda$, then $\mathcal{I}$ is not weakly $\operatorname{cf}(\lambda)$-saturated;

(d) for all $\sigma<\lambda, E_{\geq \sigma}^{\lambda^{+}} \in \mathcal{I}^{*}$.

Proof. (a) It is clear that $\mathcal{I}$ is downward closed and contains all nonstationary subsets of $\lambda^{+}$. Also, by the choice of $\vec{e}$, we know that $\lambda^{+} \notin \mathcal{I}$. Finally, since $\operatorname{cf}(\delta)=\operatorname{cf}(\lambda)$ for all $\delta \in \Delta$, and since the intersection of fewer than $\operatorname{cf}(\lambda)$-many clubs in $\lambda^{+}$is a club, we infer that $\mathcal{I}$ is $\operatorname{cf}(\lambda)$-complete.

(b) Suppose that $\tau \in \operatorname{Reg}(\lambda) \backslash\{\operatorname{cf}(\lambda)\}$ and that $\vec{A}=\left\langle A_{j} \mid j<\tau\right\rangle$ is a $\subseteq$ increasing sequence of elements from $\mathcal{I}$. We shall show that $A:=\bigcup_{j<\tau} A_{j}$ is in $\mathcal{I}$, as well. For each $j<\tau$, pick a witnessing club $D_{j}$. We claim that the club $D:=\bigcap_{j<\tau} D_{j}$ witnesses that $A \in \mathcal{I}$. To see this, let $\delta \in \Delta \cap D$ be arbitrary. Then 
$\sup \left(\operatorname{nacc}\left(e_{\delta}\right) \cap D \cap A_{j}\right)<\delta$ for all $j<\tau$. As $\operatorname{cf}(\delta) \neq \operatorname{cf}(\tau)$ and $\vec{A}$ is $\subseteq$-increasing, we infer that $\sup \left(\operatorname{nacc}\left(e_{\delta}\right) \cap D \cap A\right)<\delta$, as well.

(c) Using the fact that $\operatorname{cf}\left(\mathrm{NS}_{\mathrm{cf}(\lambda)}, \subseteq\right)<\lambda$, fix a sequence $\left\langle C^{\iota} \mid \iota<\lambda\right\rangle$ of clubs in $\operatorname{cf}(\lambda)$ such that, for every club $C$ in $\operatorname{cf}(\lambda)$, there exists $\iota<\lambda$ with $C^{\iota} \subseteq C$. For each $\iota<\lambda$ and $j<\operatorname{cf}(\lambda)$, we let $C^{\iota}(j)$ denote the unique $\alpha \in C^{\iota}$ with otp $\left(C^{\iota} \cap \alpha\right)=j$. Let $\left\langle\lambda_{j} \mid j<\operatorname{cf}(\lambda)\right\rangle$ be a strictly increasing and continuous sequence of cardinals converging to $\lambda$. For every $\iota<\lambda$, define $h^{\iota}: \lambda^{+} \rightarrow \operatorname{cf}(\lambda)$ by setting, for all $\gamma<\lambda^{+}$,

$$
h^{\iota}(\gamma):=\min \left\{j<\operatorname{cf}(\lambda) \mid \operatorname{cf}(\gamma) \leq \lambda_{C^{\iota}(j)}\right\} .
$$

Fix a surjection $\varphi: \operatorname{cf}(\lambda) \rightarrow \operatorname{cf}(\lambda)$ such that $\left|\varphi^{-1}\{i\} \cap \operatorname{nacc}(\operatorname{cf}(\lambda))\right|=\operatorname{cf}(\lambda)$ for all $i<\operatorname{cf}(\lambda)$, and then let $\Gamma_{i}^{\iota}:=\left\{\gamma<\lambda^{+} \mid \varphi\left(h^{\iota}(\gamma)\right)=i\right\}$.

We claim that there is $\iota<\lambda$ for which $\left\langle\Gamma_{i}^{\iota} \mid i<\operatorname{cf}(\lambda)\right\rangle$ is a counterexample to the weak $\operatorname{cf}(\lambda)$-saturation of $\mathcal{I}$. It is trivial to see that, for all $\iota<\lambda,\left\langle\Gamma_{i}^{\iota} \mid i<\operatorname{cf}(\lambda)\right\rangle$ is a partition of $\lambda^{+}$. Thus, it suffices to prove that there exists some $\iota<\lambda$ such that, for all $i<\operatorname{cf}(\lambda), \Gamma_{i}^{\iota} \in \mathcal{I}^{+}$. Suppose that this is not the case, and, for each $\iota<\lambda$, fix a club $D^{\iota} \subseteq \lambda^{+}$and an $i(\iota)<\operatorname{cf}(\lambda)$ such that, for every $\delta \in \Delta \cap D^{\iota}$, we have $\sup \left(\operatorname{nacc}\left(e_{\delta}\right) \cap D^{\iota} \cap \Gamma_{i(\iota)}^{\iota}\right)<\delta$. Consider the club $D:=\bigcap_{\iota<\lambda} D^{\iota}$. Pick $\delta \in \Delta$ such that $e_{\delta} \subseteq D$. Let

$$
C:=\left\{j<\operatorname{cf}(\lambda) \mid \lambda_{j} \in \operatorname{acc}^{+}\left(\left\{\operatorname{cf}(\gamma) \mid \gamma \in \operatorname{nacc}\left(e_{\delta}\right)\right\}\right)\right\},
$$

and note that $C$ is a club in $\operatorname{cf}(\lambda)$. Find $\iota<\lambda$ such that $C^{\iota} \subseteq C$. For each $j<\operatorname{cf}(\lambda)$, as $C^{\iota}(j+1) \in C$, we have $\lambda_{C^{\iota}(j+1)} \in \operatorname{acc}^{+}\left(\left\{\operatorname{cf}(\gamma) \mid \gamma \in \operatorname{nacc}\left(e_{\delta}\right)\right\}\right)$, so there exists some $\gamma \in \operatorname{nacc}\left(e_{\delta}\right)$ such that $h^{\iota}(\gamma)=j+1$. Thus, $h^{\iota}\left[\operatorname{nacc}\left(e_{\delta}\right)\right] \supseteq \operatorname{nacc}(\operatorname{cf}(\lambda)) \backslash\{0\}$, so, by the choice of $\varphi$, it follows that, for all $i<\operatorname{cf}(\lambda)$,

$$
\left|\left\{\gamma \in \operatorname{nacc}\left(e_{\delta}\right) \mid \varphi\left(h^{\iota}(\gamma)\right)=i\right\}\right|=\operatorname{cf}(\lambda)=\operatorname{otp}\left(e_{\delta}\right) .
$$

In particular, $\sup \left(\operatorname{nacc}\left(e_{\delta}\right) \cap \Gamma_{i(\iota)}^{\iota}\right)=\delta$, contradicting the fact that $e_{\delta} \subseteq D \subseteq D^{\iota}$.

(d) By the choice of $\vec{e}$, we have $E_{<\sigma}^{\lambda^{+}} \in \mathcal{I}$ for all $\sigma<\lambda$.

Next, by a standard club-swallowing trick (see the procedure before Claim 4.14.3), we may find a $C$-sequence $\vec{C}=\left\langle C_{\alpha} \mid \alpha<\lambda^{+}\right\rangle$such that

- for all $\alpha \in \operatorname{acc}\left(\lambda^{+}\right), C_{\alpha}$ is a club in $\alpha$ of order-type $<\lambda$;

- for all $\alpha \in \operatorname{acc}\left(\lambda^{+}\right)$and $\delta \in\left(\operatorname{acc}\left(C_{\alpha}\right) \cup\{\alpha\}\right) \cap \Delta$, we have $e_{\delta} \subseteq C_{\alpha}$.

Let $\operatorname{tr}:\left[\lambda^{+}\right]^{2} \rightarrow{ }^{<\omega} \lambda^{+}$denote the function derived from walking along $\vec{C}$.

Claim 4.16.2. Suppose that $\mathcal{A} \subseteq\left[\lambda^{+}\right]^{<\mathrm{cf}(\lambda)}$ is a family consisting of $\lambda^{+}$-many pairwise disjoint sets, $D$ is a club in $\lambda^{+}$, and $\Gamma \in \mathcal{I}^{+}$. Then there exist $\gamma \in D \cap \Gamma$, $a \in \mathcal{A}$, and $\epsilon<\gamma$ such that

- $\gamma<a$;

- for all $\alpha \in(\epsilon, \gamma)$ and all $\beta \in a$, we have $\gamma \in \operatorname{Im}(\operatorname{tr}(\alpha, \beta))$.

Proof. As $\Gamma \notin \mathcal{I}$, let us fix some $\delta \in \Delta$ such that $\sup \left(\operatorname{nacc}\left(e_{\delta}\right) \cap D \cap \Gamma\right)=\delta$. Pick an arbitrary $a \in \mathcal{A}$ with $a>\delta$, and put

- $\Lambda:=\sup \left\{\lambda_{2}(\delta, \beta) \mid \beta \in a\right\}$; and

- $C:=C_{\delta} \cup \bigcup_{\beta \in a} \bigcup_{\tau \in \operatorname{Im}(\operatorname{tr}(\delta, \beta))} C_{\tau}$.

As $|a|<\operatorname{cf}(\lambda)=\operatorname{cf}(\delta)$, we have $\Lambda<\delta$ and $|C|<\lambda$. Pick $\gamma \in \operatorname{nacc}\left(e_{\delta}\right) \cap D \cap \Gamma$ such that $\gamma>\Lambda$ and $\operatorname{cf}(\gamma)>|C|$. Let $\epsilon:=\max \{\Lambda, \sup (C \cap \gamma)\}$. As $\operatorname{cf}(\gamma)>|C|$, we have $\epsilon<\gamma$. We shall show that $\gamma, a$ and $\epsilon$ are as sought. 
To this end, fix arbitrary $\alpha \in(\epsilon, \gamma)$ and $\beta \in a$. We have

$$
\lambda_{2}(\delta, \beta) \leq \Lambda \leq \epsilon<\alpha<\gamma<\delta<\beta,
$$

so, by Lemma $4.6, \operatorname{tr}(\delta, \beta) \sqsubseteq \operatorname{tr}(\alpha, \beta)$. Let $\ell:=\rho_{2}(\delta, \beta)$. There are now two cases to consider.

- If $\delta \in \operatorname{nacc}\left(C_{\operatorname{tr}(\delta, \beta)(\ell-1)}\right)$, then $\sup \left(C_{\operatorname{tr}(\delta, \beta)(\ell-1)} \cap \delta\right) \leq \lambda_{2}(\delta, \beta)$ and hence

$$
[\alpha, \delta) \cap C_{\operatorname{tr}(\delta, \beta)(\ell-1)} \subseteq\left(\lambda_{2}(\delta, \beta), \delta\right) \cap C_{\operatorname{tr}(\delta, \beta)(\ell-1)}=\emptyset .
$$

Consequently, $\operatorname{tr}(\alpha, \beta)(\ell)=\min \left(C_{\operatorname{tr}(\alpha, \beta)(\ell-1)} \backslash \alpha\right)=\min \left(C_{\operatorname{tr}(\delta, \beta)(\ell-1)} \backslash \alpha\right)=\delta$. As

$$
[\alpha, \gamma) \cap C_{\operatorname{tr}(\alpha, \beta)(\ell) \subseteq(\epsilon, \gamma) \cap C=\emptyset}
$$

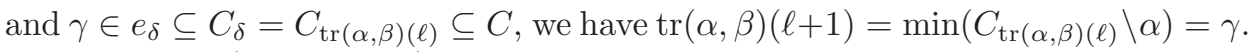

- If $\delta \in \operatorname{acc}\left(C_{\operatorname{tr}(\delta, \beta)(\ell-1)}\right)$, then

$$
[\alpha, \gamma) \cap C_{\operatorname{tr}(\delta, \beta)(\ell-1)} \subseteq(\epsilon, \gamma) \cap C=\emptyset .
$$

As $\delta \in \operatorname{acc}\left(C_{\operatorname{tr}(\delta, \beta)(\ell-1)}\right) \cap \Delta$, we have $\gamma \in e_{\delta} \subseteq C_{\operatorname{tr}(\delta, \beta)(\ell-1)} \subseteq C$. It follows that $\operatorname{tr}(\alpha, \beta)(\ell)=\min \left(C_{\operatorname{tr}(\delta, \beta)(\ell-1)} \backslash \alpha\right)=\gamma$.

We are now ready to begin verifying, in turn, that the existence of a closed witness to $\mathrm{U}\left(\lambda^{+}, \lambda^{+}, \theta, \operatorname{cf}(\lambda)\right)$ follows from each of the conditions isolated in the statement of the theorem. We begin with condition (2).

Claim 4.16.3. Suppose that $\mathcal{I}$ is not weakly $\theta$-saturated. Then there exists a closed witness to $\mathrm{U}\left(\lambda^{+}, \lambda^{+}, \theta, \operatorname{cf}(\lambda)\right)$.

Proof. Fix a function $h: \lambda^{+} \rightarrow \theta$ such that, for all $i<\theta, h^{-1}\{i\} \in I^{+}$. Derive a closed coloring $c:\left[\lambda^{+}\right]^{2} \rightarrow \theta$ as in the proof of Theorem 4.9 by letting, for all $\alpha<\beta<\lambda^{+}$,

$$
c(\alpha, \beta):=\max \{h(\tau) \mid \tau \in \operatorname{Im}(\operatorname{tr}(\alpha, \beta))\} .
$$

To show that $c$ witnesses $\mathrm{U}\left(\lambda^{+}, \lambda^{+}, \theta, \operatorname{cf}(\lambda)\right)$, it suffices to verify Clause (2) of Lemma 4.2. To this end, fix a family $\mathcal{A} \subseteq\left[\lambda^{+}\right]^{<\operatorname{cf}(\lambda)}$ consisting of $\lambda^{+}$-many pairwise disjoints sets, a club $D \subseteq \lambda^{+}$, and a color $i<\theta$. As $h^{-1}\{i+1\} \in \mathcal{I}^{+}$and $E_{\geq \operatorname{cf}(\lambda)}^{\lambda^{+}} \in \mathcal{I}^{*}$, Claim 4.16.2 provides us with $\gamma \in D \cap h^{-1}\{i+1\} \cap E_{\geq \operatorname{cf}(\lambda)}^{\lambda^{+}}, a \in \mathcal{A}$, and $\epsilon<\gamma$ such that $\gamma<a$ and, for all $\alpha \in(\epsilon, \gamma)$ and all $\beta \in a$, we have $\gamma \in \operatorname{Im}(\operatorname{tr}(\alpha, \beta))$. Then $c(\alpha, \beta) \geq h(\gamma)>i$ for all $\alpha \in(\epsilon, \gamma)$ and all $\beta \in a$, so $\gamma, a$, and $\epsilon$ witness the conclusion of Clause (2) of Lemma 4.2.

In particular, it follows from Claim 4.16.1(3) that, if $\operatorname{cf}\left(\operatorname{NS}_{\operatorname{cf}(\lambda)}, \subseteq\right)<\lambda$ and $\theta \leq \mathrm{cf}(\lambda)$, then there exists a closed witness to $\mathrm{U}\left(\lambda^{+}, \lambda^{+}, \theta, \operatorname{cf}(\lambda)\right)$.

We now turn our attention to conditions (3) and (4) from the statement of the theorem, which are taken care of by the next claim.

Claim 4.16.4. Suppose that $c:\left[\lambda^{+}\right]^{2} \rightarrow \theta$ is a coloring, $\sigma \in \operatorname{Reg}(\lambda)$, and one of the following conditions holds:

- $c$ is a somewhere-closed witness to $\mathrm{U}\left(\lambda^{+}, 2, \theta, \omega\right)$; or

- $c$ is an $E_{\geq \sigma^{+}}^{\lambda^{+}}$closed witness to $\mathrm{U}\left(\lambda^{+}, 2, \theta, 2\right)$.

Then there exists a closed witness to $\mathrm{U}\left(\lambda^{+}, \lambda^{+}, \theta, \operatorname{cf}(\lambda)\right)$.

Proof. Define $d:\left[\lambda^{+}\right]^{2} \rightarrow \theta$ by setting, for all $\alpha<\beta<\lambda^{+}$,

$$
d(\alpha, \beta):=\max \left\{c(\delta, \gamma) \mid(\delta, \gamma) \in[\operatorname{Im}(\operatorname{tr}(\alpha, \beta))]^{2}\right\},
$$

provided that the set is nonempty, and $d(\alpha, \beta):=0$, otherwise. 
We claim that $d$ is as desired. To see that $d$ is closed, suppose that $\beta<\lambda^{+}, i<\theta$, and $A \subseteq D_{\leq i}^{d}(\beta)$, with $\eta:=\sup (A)$ smaller than $\beta$. To show that $\eta \in D_{<i}^{d}(\beta)$, fix $\alpha \in A$ above $\lambda_{2}(\eta, \beta)$. By Lemma 4.6, $\operatorname{Im}(\operatorname{tr}(\eta, \beta)) \subseteq \operatorname{Im}(\operatorname{tr}(\alpha, \beta))$, and hence, by the definition of $d$, we have $d(\eta, \beta) \leq d(\alpha, \beta) \leq i$.

To see that $d$ witnesses $\mathrm{U}\left(\lambda^{+}, \lambda^{+}, \theta, \operatorname{cf}(\lambda)\right)$, it suffices to verify Clause (2) of Lemma 4.2. To this end, suppose that $\mathcal{A} \subseteq\left[\lambda^{+}\right]<\operatorname{cf}(\lambda)$ is a family consisting of $\lambda^{+}$-many pairwise disjoint sets, $D$ is a club in $\lambda^{+}$, and $i<\theta$. We shall prove that there exist $\zeta \in D, a \in \mathcal{A}$, and $\epsilon^{*}<\zeta$ for which

- $\zeta<a$;

- for all $\alpha \in\left(\epsilon^{*}, \zeta\right)$ and all $\beta \in a$, we have $d(\alpha, \beta)>i$.

Let $\Gamma$ be the set of $\gamma \in E_{\geq \sigma}^{\lambda^{+}}$for which there exist $a \in \mathcal{A}$ and $\epsilon<\gamma$ such that

- $\gamma<a$;

- for all $\beta \in a$ and $\alpha \in(\epsilon, \gamma)$, we have $\gamma \in \operatorname{Im}(\operatorname{tr}(\alpha, \beta))$.

By Claim 4.16.2 and Claim 4.16.1(4), $\Gamma$ is stationary. For each $\gamma \in \Gamma$, pick $a_{\gamma} \in \mathcal{A}$ and $\epsilon_{\gamma}<\gamma$ witnessing $\gamma \in \Gamma$. Fix a stationary subset $\Gamma^{\prime} \subseteq \Gamma$ on which the map $\gamma \mapsto \epsilon_{\gamma}$ is constant, with value, say, $\epsilon$. Now, let $S$ be the set of $\delta<\lambda^{+}$for which there exist $\gamma \in \Gamma^{\prime}$ and $\varepsilon<\delta$ such that

- $\delta<\gamma$;

- for all $\zeta \in(\varepsilon, \delta)$, we have $c(\zeta, \gamma)>i$.

We claim that $S$ is stationary. To see this, consider the following two cases.

- If $c$ is a somewhere-closed witness to $\mathrm{U}\left(\lambda^{+}, 2, \theta, \omega\right)$, then by the implication $(1) \Longrightarrow(2)$ of Lemma 4.2, we infer that $S$ is stationary.

- If $c$ is a $E_{>\sigma^{+}}^{\lambda^{+}}$-closed witness to $\mathrm{U}\left(\lambda^{+}, 2, \theta, 2\right)$, then repeating the proof of the implication $(\overline{1}) \Longrightarrow(2)$ of Lemma 4.2 in the current setting implies that, furthermore, $S \cap \Gamma^{\prime}$ is stationary.

For each $\delta \in S$, pick $\gamma_{\delta} \in \Gamma^{\prime}$ and $\varepsilon_{\delta}<\delta$ witnessing $\delta \in S$. Fix a stationary subset $S^{\prime} \subseteq S$ on which the map $\delta \mapsto \varepsilon_{\delta}$ is constant, with value, say, $\varepsilon$. Finally, let $Z$ be the set of $\zeta \in E_{\geq \operatorname{cf}(\lambda)}^{\lambda^{+}}$for which there exist $\delta \in S^{\prime}$ and $\eta<\zeta$ such that

- $\zeta<\delta$;

- for all $\alpha \in(\eta, \zeta)$, we have $\zeta \in \operatorname{Im}\left(\operatorname{tr}\left(\alpha, \gamma_{\delta}\right)\right)$.

By Claim 4.16.2 and Claim 4.16.1(4), $Z$ is stationary, so we may find $\zeta \in Z \cap D$ above $\max \{\epsilon, \varepsilon\}$. Pick $\delta \in S^{\prime}$ and $\eta<\zeta$ above $\max \{\epsilon, \varepsilon\}$ such that $\delta>\zeta$ and, for all $\alpha \in(\eta, \zeta)$, we have $\zeta \in \operatorname{Im}\left(\operatorname{tr}\left(\alpha, \gamma_{\delta}\right)\right)$. Set $a:=a_{\gamma_{\delta}}$ and $\epsilon^{*}:=\eta$. We claim that $\zeta$, $a$, and $\epsilon^{*}$ are as desired. To this end, let $\alpha \in\left(\epsilon^{*}, \zeta\right)$ and $\beta \in a$ be arbitrary. Then

$$
\max \{\epsilon, \varepsilon\}<\eta<\alpha<\zeta<\delta<\gamma_{\delta}<\beta \text {. }
$$

As $\gamma_{\delta} \in \Gamma^{\prime}, \beta \in a_{\gamma_{\delta}}$, and $\alpha \in\left(\epsilon, \gamma_{\delta}\right)$, we have $\gamma_{\delta} \in \operatorname{Im}(\operatorname{tr}(\alpha, \beta))$. Next, as $\alpha \in(\eta, \zeta)$, we have $\zeta \in \operatorname{Im}\left(\operatorname{tr}\left(\alpha, \gamma_{\delta}\right)\right)$, and, consequently, $\zeta \in \operatorname{Im}(\operatorname{tr}(\alpha, \beta))$. Finally, as $\delta \in S^{\prime}$ and $\zeta \in(\varepsilon, \delta)$, we infer that $c\left(\zeta, \gamma_{\delta}\right)>i$. Altogether, we obtain $d(\alpha, \beta)>i$, as desired.

We end the uncountable cofinality case of the proof by addressing condition (1).

Claim 4.16.5. Suppose that there exists no closed witness to $\mathrm{U}\left(\lambda^{+}, \lambda^{+}, \theta, \operatorname{cf}(\lambda)\right)$. Then $\operatorname{Refl}\left(<\operatorname{cf}(\lambda), \lambda^{+}\right)$holds.

Proof. By Corollary 4.13, $\theta \neq \operatorname{cf}(\lambda)$. So, by Claims 4.16.1 and 4.16.3, it follows that $\mathcal{I}$ is a $\operatorname{cf}(\lambda)$-complete ideal which is weakly $\theta$-saturated and $\theta$-indecomposable. 
But then by [Eis10, Theorem 2(4)], $\operatorname{Refl}\left(<\operatorname{cf}(\lambda), S^{*}\right)$ holds for $S^{*}:=E_{>\theta}^{\lambda^{+}} \cap E_{\neq \operatorname{cf}(\lambda)}^{\lambda^{+}}$. In addition, by Claim 4.16.4, there exists no closed witness to $\mathrm{U}\left(\lambda^{+}, \lambda^{+}, \theta, \omega\right)$, and hence by Theorem 4.14, for every stationary $S \subseteq \lambda^{+}$, we know that $\operatorname{Tr}(S) \cap S^{*}$ is stationary. Consequently, $\operatorname{Refl}\left(<\operatorname{cf}(\lambda), \lambda^{+}\right)$holds.

Case 2: Countable cofinality. Assume now that $\operatorname{cf}(\lambda)=\omega$. Let $\chi:=\theta^{+}$ and $\Delta:=E_{\chi}^{\lambda^{+}}$. By a result of Eisworth [Eis10, $\left.\S 5\right]$, we obtain a strictly increasing sequence of regular cardinals $\left\langle\lambda_{m} \mid m<\omega\right\rangle$ that converges to $\lambda$ and two matrices, $\vec{C}=\left\langle C_{\alpha}^{m} \mid \alpha<\lambda^{+}, m<\omega\right\rangle$ and $\vec{e}=\left\langle e_{\delta}^{m} \mid \delta \in \Delta, m<\omega\right\rangle$, such that

- for all $m<\omega, C_{0}^{m}=\emptyset$;

- for all $\alpha<\lambda^{+}$and $m<\omega, C_{\alpha+1}^{m}=\{\alpha\}$;

- for all $\delta \in \Delta,\left\langle e_{\delta}^{m} \mid m<\omega\right\rangle$ is a $\subseteq$-increasing sequence of club subsets of $\delta$;

- for all $\alpha \in \operatorname{acc}\left(\lambda^{+}\right),\left\langle C_{\alpha}^{m} \mid m<\omega\right\rangle$ is a $\subseteq$-increasing sequence of club subsets of $\alpha$;

- for all $\alpha \in \operatorname{acc}\left(\lambda^{+}\right)$and $m<\omega,\left|C_{\alpha}^{m}\right| \leq \max \left\{\lambda_{m}, \operatorname{cf}(\alpha)\right\}$;

- for all $\alpha \in \operatorname{acc}\left(\lambda^{+}\right), m<\omega$, and $\delta \in\left(\operatorname{acc}\left(C_{\alpha}^{m}\right) \cup\{\alpha\}\right) \cap \Delta, e_{\delta}^{m} \subseteq C_{\alpha}^{m}$;

- for every club $D$ in $\lambda^{+}$, there exists $\delta \in \Delta$ such that $\sup \left(e_{\delta}^{m} \cap D \cap E_{>\lambda_{m}}^{\delta}\right)=\delta$ for all $m<\omega$.

Define $\mathcal{I} \subseteq \mathcal{P}\left(\lambda^{+}\right)$by letting $A \in \mathcal{P}\left(\lambda^{+}\right)$be in $\mathcal{I}$ iff there exists a club $D \subseteq \lambda^{+}$such that for every $\delta \in \Delta \cap D$, for a tail of $m<\omega$, we have $\sup \left(e_{\delta}^{m} \cap D \cap E_{>\lambda_{m}}^{\delta} \cap A\right)<\delta$.

Claim 4.16.6. I satisfies all of the following properties:

(a) $\mathcal{I}$ is a proper ideal over $\lambda^{+}$, extending $\mathrm{NS}_{\lambda^{+}}$;

(b) $\mathcal{I}$ is $\tau$-indecomposable for all $\tau \in \operatorname{Reg}(\lambda) \backslash\{\omega, \chi\}$;

(c) for all $\sigma<\lambda, E_{\geq \sigma}^{\lambda^{+}} \in \mathcal{I}^{*}$.

Proof. (b) Suppose that $\tau \in \operatorname{Reg}(\lambda) \backslash\{\omega, \chi\}$ and that $\vec{A}=\left\langle A_{j} \mid j<\tau\right\rangle$ is a $\subseteq$ increasing sequence of elements from $\mathcal{I}$. We shall show that $A:=\bigcup_{j<\tau} A_{j}$ is in $\mathcal{I}$, as well. For each $j<\tau$, pick a witnessing club $D_{j}$. We claim that the club $D:=\bigcap_{j<\tau} D_{j}$ witnesses that $A \in \mathcal{I}$. To see this, let $\delta \in \Delta \cap D$ be arbitrary. For each $m<\omega$ such that $\sup \left(e_{\delta}^{m} \cap D \cap E_{>\lambda_{m}}^{\delta} \cap A\right)=\delta$, as $\vec{A}$ is $\subseteq$-increasing and $\operatorname{cf}(\delta) \neq \operatorname{cf}(\tau)$, we may find $j_{m}<\tau$ such that $\sup \left(e_{\delta}^{m} \cap D \cap E_{>\lambda_{m}}^{\delta} \cap A_{j_{m}}\right)=\delta$. As $\vec{A}$ is $\subseteq$-increasing and $\operatorname{cf}(\tau)>\omega$, it follows that there exists a large enough $j<\omega$ such that, for all $m<\omega$, if $\sup \left(e_{\delta}^{m} \cap D \cap E_{>\lambda_{m}}^{\delta} \cap A\right)=\delta$, then $\sup \left(e_{\delta}^{m} \cap D \cap E_{>\lambda_{m}}^{\delta} \cap A_{j}\right)=\delta$. But $D \subseteq D_{j}$, and hence $\sup \left(e_{\delta}^{m} \cap D \cap E_{>\lambda_{m}}^{\delta} \cap A_{j}\right)<\delta$ for a tail of $m<\omega$. So, $\sup \left(e_{\delta}^{m} \cap D \cap E_{>\lambda_{m}}^{\delta} \cap A\right)=\delta$ for a tail of $m<\omega$.

For each $m<\omega$, let $\operatorname{tr}^{m}(\cdot, \cdot), \rho_{2}^{m}(\cdot, \cdot)$ and $\lambda_{2}^{m}(\cdot, \cdot)$ denote the respective characteristic functions derived from walking along the $C$-sequence $\left\langle C_{\alpha}^{m} \mid \alpha<\lambda^{+}\right\rangle$. Note that for all $\alpha<\beta<\lambda^{+}$, there is $n<\omega$ such that, for every integer $m \geq n$, we have $\operatorname{tr}^{m}(\alpha, \beta)=\operatorname{tr}^{n}(\alpha, \beta)$ (cf. [Rin12, p. 1094]).

Claim 4.16.7. Suppose that $\mathcal{A} \subseteq\left[\lambda^{+}\right]<\omega$ is a family consisting of $\lambda^{+}$-many pairwise disjoint sets, $D$ is a club in $\lambda^{+}$, and $\Gamma \in \mathcal{I}^{+}$. Then there exist $\gamma \in D \cap \Gamma, a \in \mathcal{A}$, $\epsilon<\gamma$, and $k<\omega$ such that

- $\gamma<a$;

- for all $\alpha \in(\epsilon, \gamma)$ and all $\beta \in a$, we have $\gamma \in \operatorname{Im}\left(\operatorname{tr}^{k}(\alpha, \beta)\right)$. 
Proof. Since $\Gamma \notin \mathcal{I}$, we may fix $\delta \in \Delta$ such that $\sup \left(e_{\delta}^{m} \cap D \cap E_{>\lambda_{m}}^{\delta} \cap \Gamma\right)=\delta$ for cofinally many $m<\omega$. Fix an arbitrary $a \in \mathcal{A}$ with $a>\delta$. Since $a$ is finite, we may find an $n<\omega$ large enough so that, for every $\beta \in a$ and every integer $m \geq n$, we have $\operatorname{tr}^{m}(\delta, \beta)=\operatorname{tr}^{n}(\delta, \beta)$.

Consider the finite set $T:=\{\delta\} \cup \bigcup_{\beta \in a} \operatorname{Im}\left(\operatorname{tr}^{n}(\delta, \beta)\right)$, and then find an integer $k>n$ such that $\max \{\operatorname{cf}(\tau) \mid \tau \in T\} \leq \lambda_{k}$ and $\sup \left(e_{\delta}^{k} \cap D \cap E_{>\lambda_{k}}^{\delta} \cap A\right)=\delta$. Finally, pick $\gamma \in e_{\delta}^{k} \cap D \cap E_{>\lambda_{k}}^{\delta}$ above $\Lambda:=\sup \left\{\lambda_{2}^{k}(\delta, \beta) \mid \beta \in a\right\}$. Let $C:=\bigcup\left\{C_{\tau}^{k} \mid\right.$ $\tau \in T\}$. For all $\tau \in T$, we have $\left|C_{\tau}^{k}\right| \leq \max \left\{\lambda_{k}, \operatorname{cf}(\tau)\right\}=\lambda_{k}<\operatorname{cf}(\gamma)$, and hence $\epsilon:=\max \{\Lambda, \sup (C \cap \gamma)\}$ is less than $\gamma$.

We claim that $\gamma, a, \epsilon$, and $k$ are as desired. We clearly have $\gamma<a$. To finish, fix an arbitrary $\beta \in a$ and $\alpha \in(\epsilon, \gamma)$. We have

$$
\lambda_{2}^{k}(\delta, \beta) \leq \Lambda \leq \epsilon<\alpha<\gamma<\delta<\beta,
$$

so, by Lemma $4.6, \operatorname{tr}^{k}(\delta, \beta) \sqsubseteq \operatorname{tr}^{k}(\alpha, \beta)$. Set $\ell:=\rho_{2}^{k}(\delta, \beta)$. There are now two cases to consider.

- If $\delta \in \operatorname{nacc}\left(C_{\operatorname{tr}^{k}(\delta, \beta)(\ell-1)}^{k}\right)$, then, since

$$
[\alpha, \delta) \cap C_{\operatorname{tr}^{k}(\delta, \beta)(\ell-1)}^{k} \subseteq\left(\lambda_{2}^{k}(\delta, \beta), \delta\right) \cap C_{\operatorname{tr}^{k}(\delta, \beta)(\ell-1)}^{k}=\emptyset,
$$

we have $\operatorname{tr}^{k}(\alpha, \beta)(\ell)=\min \left(C_{\operatorname{tr}^{k}(\alpha, \beta)(\ell-1)}^{k} \backslash \alpha\right)=\min \left(C_{\operatorname{tr}^{k}(\delta, \beta)(\ell-1)}^{k} \backslash \alpha\right)=\delta$. It follows that $\gamma \in e_{\delta}^{k} \subseteq C_{\delta}^{k} \subseteq C$, so

$$
[\alpha, \gamma) \cap C_{\operatorname{tr}^{k}(\alpha, \beta)(\ell)}^{k} \subseteq(\epsilon, \gamma) \cap C=\emptyset,
$$

and hence $\operatorname{tr}^{k}(\alpha, \beta)(\ell+1)=\min \left(C_{\operatorname{tr}^{k}(\alpha, \beta)(\ell)}^{k} \backslash \alpha\right)=\gamma$.

- If $\delta \in \operatorname{acc}\left(C_{\operatorname{tr}^{k}(\delta, \beta)(\ell-1)}^{k}\right)$, then, since $\delta \in \Delta$, we observe that $\gamma \in e_{\delta}^{k} \subseteq$ $C_{\operatorname{tr}^{k}(\delta, \beta)(\ell-1)}^{k} \subseteq C$. It follows that $[\alpha, \gamma) \cap C_{\operatorname{tr}^{k}(\delta, \beta)(\ell-1)}^{k}=\emptyset$, and hence $\operatorname{tr}^{k}(\alpha, \beta)(\ell)=$ $\min \left(C_{\operatorname{tr}^{k}(\delta, \beta)(\ell-1)}^{k} \backslash \alpha\right)=\gamma$.

We now show that the existence of a closed witness to $\mathrm{U}\left(\lambda^{+}, \lambda^{+}, \theta, \omega\right)$ follows from each of the hypotheses identified in the statement of the theorem. Note first that condition (2) is trivially taken care of, as there are no infinite cardinals strictly less than $\omega=\operatorname{cf}(\lambda)$. The next claim will deal with conditions (3) and (4).

Claim 4.16.8. Suppose that $c:\left[\lambda^{+}\right]^{2} \rightarrow \theta$ is a coloring, $\sigma \in \operatorname{Reg}(\lambda)$, and one of the following two conditions holds:

- $c$ is a somewhere-closed witness to $\mathrm{U}\left(\lambda^{+}, 2, \theta, \omega\right)$; or

- $c$ is a $E_{\geq \sigma^{+}}^{\lambda^{+}}$closed witness to $\mathrm{U}\left(\lambda^{+}, 2, \theta, 2\right)$.

Then there exists a closed witness to $\mathrm{U}\left(\lambda^{+}, \lambda^{+}, \theta, \omega\right)$.

Proof. Define $d:\left[\lambda^{+}\right]^{2} \rightarrow \theta$ by setting, for all $\alpha<\beta<\lambda^{+}$,

$$
d(\alpha, \beta):=\max \left\{c(\zeta, \gamma) \mid(\zeta, \gamma) \in\left[\bigcup_{m<\omega} \operatorname{Im}\left(\operatorname{tr}^{m}(\alpha, \beta)\right)\right]^{2}\right\}
$$

provided that the set is nonempty, and $d(\alpha, \beta):=0$, otherwise.

We claim that $d$ is as desired. To see that $d$ is closed, suppose that $\beta<\lambda^{+}$, $i<\theta$, and $A \subseteq D_{\leq i}^{d}(\beta)$, with $\eta:=\sup (A)$ smaller than $\beta$. To show that $\eta \in D_{\leq i}^{d}(\beta)$, fix $n<\omega$ large enough so that $\left\{\operatorname{tr}^{m}(\eta, \beta) \mid m<\omega\right\}=\left\{\operatorname{tr}^{m}(\eta, \beta) \mid m<n\right\}$, and then fix $\alpha \in A$ above $\max _{m<n} \lambda_{2}^{m}(\eta, \beta)$. By Lemma 4.6, $\bigcup_{m<n} \operatorname{Im}\left(\operatorname{tr}^{m}(\eta, \beta)\right) \subseteq$ 
$\bigcup_{m<\omega} \operatorname{Im}^{m}(\operatorname{tr}(\alpha, \beta))$, and hence, by the definition of $d$, we have $d(\eta, \beta) \leq d(\alpha, \beta) \leq$ $i$.

To see that $d$ witnesses $\mathrm{U}\left(\lambda^{+}, \lambda^{+}, \theta, \omega\right)$, it suffices to verify Clause (2) of Lemma 4.2. To this end, suppose that $\mathcal{A} \subseteq\left[\lambda^{+}\right]^{<\omega}$ is a family consisting of $\lambda^{+}$-many pairwise disjoint sets, $D$ is a club in $\lambda^{+}$, and $i<\theta$. We shall prove that there exist $\zeta \in D$, $a \in \mathcal{A}$, and $\epsilon^{*}<\zeta$ for which

- $\zeta<a$;

- for all $\alpha \in\left(\epsilon^{*}, \zeta\right)$ and all $\beta \in a$, we have $d(\alpha, \beta)>i$.

Let $\Gamma$ be the set of $\gamma \in E_{\geq \sigma}^{\lambda^{+}}$for which there exist $a \in \mathcal{A}, \epsilon<\gamma$, and $k<\omega$ such that

- $\gamma<a$;

- for all $\beta \in a$ and $\alpha \in(\epsilon, \gamma)$, we have $\gamma \in \operatorname{Im}\left(\operatorname{tr}^{k}(\alpha, \beta)\right)$.

By Claim 4.16.7 and Claim 4.16.6(3), $\Gamma$ is stationary. For each $\gamma \in \Gamma$, pick $a_{\gamma} \in \mathcal{A}$, $\epsilon_{\gamma}<\gamma$ and $k_{\gamma}<\omega$ witnessing that $\gamma \in \Gamma$. Fix a stationary subset $\Gamma^{\prime} \subseteq \Gamma$ on which the map $\gamma \mapsto\left(\epsilon_{\gamma}, k_{\gamma}\right)$ is constant, with value, say, $(\epsilon, k)$.

Now, let $S$ be the set of $\varsigma<\lambda^{+}$for which there exist $\gamma \in \Gamma^{\prime}$ and $\varepsilon<\varsigma$ such that

- $\varsigma<\gamma$;

- for all $\zeta \in(\varepsilon, \varsigma)$, we have $c(\zeta, \gamma)>i$.

We claim that $S$ is stationary. There are two cases to consider.

- If $c$ is a somewhere-closed witness to $\mathrm{U}\left(\lambda^{+}, 2, \theta, \omega\right)$, then, by the implication $(1) \Longrightarrow(2)$ of Lemma 4.2 , we infer that $S$ is stationary.

- If $c$ is a $E_{>\sigma^{-}}^{\lambda^{+}}$-closed witness to $\mathrm{U}\left(\lambda^{+}, 2, \theta, 2\right)$, then repeating the proof of the implication $(\overline{1}) \Longrightarrow(2)$ of Lemma 4.2 in the current setting implies that, furthermore, $S \cap \Gamma^{\prime}$ is stationary.

For each $\varsigma \in S$, pick $\gamma_{\varsigma} \in \Gamma^{\prime}$ and $\varepsilon_{\varsigma}<\varsigma$ witnessing that $\varsigma \in S$. Fix a stationary subset $S^{\prime} \subseteq S$ on which the map $\varsigma \mapsto \varepsilon_{\varsigma}$ is constant, with value, say, $\varepsilon$.

Subclaim 4.16.8.1. There exist $\zeta \in D, \varsigma \in S^{\prime}, \eta<\zeta$ and $l<\omega$ such that

- $a_{\gamma_{\varsigma}}>\gamma_{\varsigma}>\varsigma>\zeta>\max \{\epsilon, \varepsilon\}$;

- for all $\beta \in a_{\gamma_{\varsigma}}$ and $\alpha \in(\eta, \zeta)$, we have $\zeta \in \operatorname{Im}\left(\operatorname{tr}^{l}(\alpha, \beta)\right)$.

Proof. The proof is nearly identical to that of Claim 4.16.7. Fix $\delta \in \Delta$ above $\max \{\epsilon, \varepsilon\}$ such that $\sup \left(e_{\delta}^{m} \cap D \cap E_{>\lambda_{m}}^{\delta}\right)=\delta$ for all $m<\omega$. Fix $\varsigma \in S^{\prime}$ above $\delta$, and let $a:=a_{\gamma_{\varsigma}}$. Find $n<\omega$ large enough so that, for every $\beta \in a$ and every integer $m \geq n$, we have $\operatorname{tr}^{m}(\delta, \beta)=\operatorname{tr}^{n}(\delta, \beta)$. Let $T:=\{\delta\} \cup \bigcup_{\beta \in a} \operatorname{Im}\left(\operatorname{tr}^{n}(\delta, \beta)\right)$, and find an integer $l>n$ large enough so that, for all $\tau \in T$, we have $\operatorname{cf}(\tau) \leq \lambda_{l}$. Let $\Lambda:=\sup \left\{\lambda_{2}^{l}(\delta, \beta) \mid \beta \in a\right\}$, and pick $\zeta \in e_{\delta}^{l} \cap D \cap E_{>\lambda_{l}}^{\lambda^{+}}$above $\max \{\Lambda, \epsilon, \varepsilon\}$. Let $C:=\bigcup\left\{C_{\tau}^{l} \mid \tau \in T\right\}$. For all $\tau \in T$, we have $\left|C_{\tau}^{l}\right| \leq \max \left\{\lambda_{l}, \operatorname{cf}(\tau)\right\}=\lambda_{l}<\operatorname{cf}(\zeta)$, and hence $\eta:=\max \{\Lambda, \sup (C \cap \zeta)\}$ is less than $\zeta$.

We claim that $\zeta, \varsigma, \eta$, and $l$ are as desired. The first requirement is clearly satisfied. To verify the second, fix an arbitrary $\beta \in a$ and $\alpha \in(\eta, \zeta)$. We have

$$
\lambda_{2}^{l}(\delta, \beta) \leq \Lambda \leq \eta<\alpha<\zeta<\delta<\varsigma<\gamma_{\varsigma}<\beta,
$$

so, by Lemma 4.6, $\rho_{2}^{l}(\delta, \beta) \sqsubseteq \rho_{2}^{l}(\alpha, \beta)$. Let $\ell:=\rho_{2}^{l}(\delta, \beta)$. Then, as in the proof of Claim 4.16.7, we infer that $\zeta \in\left\{\operatorname{tr}^{l}(\alpha, \beta)(\ell), \operatorname{tr}^{l}(\alpha, \beta)(\ell+1)\right\}$.

Let $\zeta, \varsigma, \eta$, and $l$ be given by the preceding subclaim. Let $\gamma:=\gamma_{\varsigma}, a:=a_{\gamma}$, and $\epsilon^{*}:=\max \{\epsilon, \varepsilon, \eta\}$. We claim that $\zeta, a$, and $\epsilon^{*}$ are as sought. To prove this, let 
$\alpha \in\left(\epsilon^{*}, \zeta\right)$ and $\beta \in a$ be arbitrary. As $\gamma \in \Gamma^{\prime}, \beta \in a=a_{\gamma}$, and $\alpha \in(\epsilon, \gamma)$, we have $\gamma \in \operatorname{Im}\left(\operatorname{tr}^{k}(\alpha, \beta)\right)$. As $\alpha \in(\eta, \zeta)$, we have $\zeta \in \operatorname{Im}\left(\operatorname{tr}^{l}(\alpha, \beta)\right)$. Finally, as $\varsigma \in S^{\prime}$ and $\zeta \in(\varepsilon, \varsigma)$, we have $c(\zeta, \gamma)>i$. Altogether, we obtain $d(\alpha, \beta)>i$, as desired.

We now finish the proof of the countable cofinality case and hence the theorem by disposing with condition (1).

Claim 4.16.9. Suppose that there exists no closed witness to $\mathrm{U}\left(\lambda^{+}, \lambda^{+}, \theta, \omega\right)$. Then $\operatorname{Refl}\left(<\omega, \lambda^{+}\right)$holds.

Proof. The proof of Claim 4.16.3 makes it clear that Claim 4.16.7 implies the existence of a closed witness to $\mathrm{U}\left(\lambda^{+}, \lambda^{+}, \theta, \omega\right)$, provided that $\mathcal{I}$ is not weakly $\theta$ saturated. Consequently, $\mathcal{I}$ is weakly $\theta$-saturated. By Corollary 4.13, $\theta \neq \omega$. Altogether, $\theta \in \operatorname{Reg}(\lambda) \backslash\{\omega, \chi\}$, so, by Claim 4.16.6, $\mathcal{I}$ is an ideal that is weakly $\theta$-saturated and $\theta$-indecomposable. It then follows from [Eis10, Theorem 2(4)] that $\operatorname{Refl}\left(<\omega, S^{*}\right)$ holds, where $S^{*}:=E_{\geq \theta}^{\lambda^{+}} \cap E_{\neq \omega}^{\lambda^{+}}$. In addition, by Theorem 4.14, for every stationary $S \subseteq \lambda^{+}$, we know that $\operatorname{Tr}(S) \cap S^{*}$ is stationary. Therefore, $\operatorname{Refl}\left(<\omega, \lambda^{+}\right)$ holds.

4.5. Inaccessible cardinals. We begin this subsection by noting the following result. It follows immediately from the proof of Theorem 4.14, so we do not provide a separate proof here.

Proposition 4.17. Suppose that $\kappa$ is an inaccessible cardinal, $\theta, \chi \in \operatorname{Reg}(\kappa)$, and there exists a stationary $S \subseteq E_{\chi}^{\kappa}$ such that $(S)$ holds and

$$
\sup \left\{\nu<\kappa \mid \operatorname{Tr}(S) \cap E_{\nu}^{\kappa} \text { is stationary }\right\}<\kappa .
$$

Then there exists a closed witness to $\mathrm{U}(\kappa, \kappa, \theta, \chi)$.

Our last result of this section, similarly to Theorem 4.14, provides an improvement to the implication $(3) \Longrightarrow(4)$ from Corollary 4.10 , this time in the context of inaccessible cardinals.

Theorem 4.18. Suppose that $\kappa$ is an inaccessible cardinal, $\theta, \chi \in \operatorname{Reg}(\kappa)$, and there exists a stationary $S \subseteq E_{\geq \chi}^{\kappa}$ that does not reflect at any inaccessible cardinal. Then there exists a closed witness to $\mathrm{U}(\kappa, \kappa, \theta, \chi)$.

Proof. By Corollary 4.14, we may assume that, for every stationary $T \subseteq E_{\geq \chi}^{\kappa}$, the set $\operatorname{Tr}(T) \cap E_{>\theta}^{\kappa}$ is stationary. We begin by isolating stationary sets as in the statement of the theorem that are slightly better-behaved.

Claim 4.18.1. There exist regular cardinals $\sigma, \tau$ with $\max \left\{\aleph_{1}, \chi, \theta\right\} \leq \sigma<\tau<\kappa$ and stationary subsets $S, S^{0}$ of $\kappa$ such that

- $S \subseteq E_{\sigma}^{\kappa} \cap$ Card, and $S$ does not reflect at inaccessibles;

- $S^{0} \subseteq E_{\tau}^{\kappa}$, and $S^{0}$ does not reflect at inaccessibles.

Proof. By the hypothesis of the theorem, we can fix a stationary $T \subseteq E_{\geq \chi}^{\kappa}$ such that $T$ does not reflect at inaccessibles. Then $\operatorname{Tr}(T) \cap E_{>\theta}^{\kappa}$ is a stationary set consisting of singular ordinals, so Fodor's lemma entails the existence of a cardinal $\sigma \in \operatorname{Reg}(\kappa) \backslash \theta$ for which $\operatorname{Tr}(T) \cap E_{\sigma}^{\kappa}$ is stationary. Since Card $\cap \kappa$ is a club in the inaccessible $\kappa$, $S:=\operatorname{Tr}(T) \cap \operatorname{Card} \cap E_{\sigma}^{\kappa}$ is a stationary subset of $E_{>\chi}^{\kappa}$. As $\operatorname{Tr}(S) \subseteq \operatorname{Tr}(T)$, we can repeat the process to find $\tau \in \operatorname{Reg}(\kappa)$ such that $S^{0}:=\operatorname{Tr}(S) \cap E_{\tau}^{\kappa}$ is stationary. Then $\tau>\sigma>\chi \geq \aleph_{0}, \sigma \geq \theta$ and $\operatorname{Tr}\left(S^{0}\right) \subseteq \operatorname{Tr}(S) \subseteq \operatorname{Tr}(T)$, so $\sigma, \tau, S$, and $S^{0}$ are as sought. 
Let $\sigma, \tau, S$, and $S^{0}$ be given by the preceding claim. By [Hof13, Theorem 2.1.1], there exists a sequence $\left\langle e_{\delta} \mid \delta \in S\right\rangle$ such that

- for all $\delta \in S, e_{\delta}$ is a club in $\delta$ of order type $\sigma$;

- for all $\delta \in S,\left\langle\operatorname{cf}(\gamma) \mid \gamma \in \operatorname{nacc}\left(e_{\delta}\right)\right\rangle$ is strictly increasing and converges to $\delta$;

- for every club $D \subseteq \kappa$, there exists $\delta \in S$ with $e_{\delta} \subseteq D$.

Define $\mathcal{I} \subseteq \mathcal{P}(\kappa)$ by letting $A \in \mathcal{P}(\kappa)$ be in $\mathcal{I}$ iff there exists a club $D \subseteq \kappa$ such that for every $\delta \in S \cap \operatorname{acc}(D), \sup \left(\operatorname{nacc}\left(e_{\delta}\right) \cap D \cap A\right)<\delta$.

Claim 4.18.2. I satisfies the following two conditions:

(1) $\mathcal{I}$ is a $\sigma$-complete proper ideal over $\kappa$, extending $\mathrm{NS}_{\kappa}$;

(2) $\mathcal{I}$ is not weakly $\theta$-saturated.

Proof. Clause (1) is straightforward to verify. To see that Clause (2) holds, we shall want to appeal to [She94, Claim 3.3]. For each $\delta \in S$, let $I_{\delta}:=\left\{A \subseteq e_{\delta} \mid\right.$ $\left.\sup \left(\operatorname{nacc}\left(e_{\delta}\right) \cap A\right)<\delta\right\}$, so that $I_{\delta}$ is a $\sigma$-complete and $\tau$-indecomposable ideal over $e_{\delta}$. Trivially, $\sup _{\delta \in S}\left|e_{\delta}\right|^{+}\left\langle\kappa\right.$. Setting $\bar{C}:=\left\langle e_{\delta} \mid \delta \in S\right\rangle$ and $\bar{I}:=\left\langle I_{\delta} \mid \delta \in S\right\rangle$, and recalling [She94, Definition 3.1], it is evident that the ideal $\operatorname{id}_{p}(\bar{C}, \bar{I})$ is equal to our proper ideal $\mathcal{I}$. Now, since $S^{0}$ is a stationary subset of $E_{\tau}^{\kappa}$ that does not reflect at inaccessibles, Case $(\beta)(a)$ of [She94, Claim 3.3] entails the existence of a partition of $\kappa$ into $\tau$-many $\mathcal{I}$-positive sets. In particular, since $\tau>\theta, \mathcal{I}$ is not weakly $\theta$-saturated.

Using the preceding claim, fix a surjection $h: \kappa \rightarrow \theta$ such that $h^{-1}\{i\} \in \mathcal{I}^{+}$for all $i<\theta$. Next, using [Hof13, Proposition 4.3.1] and the fact that $S \subseteq$ Card and $S$ does not reflect at inaccessibles, fix a $C$-sequence $\vec{C}=\left\langle C_{\alpha} \mid \alpha<\kappa\right\rangle$ such that

- for all $\alpha \in \operatorname{Reg}(\kappa), C_{\alpha}$ is a club in $\alpha$ disjoint from $S$;

- for all $\alpha \in \operatorname{acc}(\kappa) \backslash \operatorname{Reg}(\kappa), C_{\alpha}$ is a club in $\alpha$ satisfying:

$-\left|C_{\alpha}\right|<\min \left(C_{\alpha}\right)$

$-\left|C_{\alpha}\right| \leq \max \{\sigma, \operatorname{cf}(\alpha)\}$

- for all $\delta \in\left(C_{\alpha} \cup\{\alpha\}\right) \cap S, \sup \left(e_{\delta} \backslash C_{\alpha}\right)<\delta$.

We shall walk along $\vec{C}$. Derive a closed coloring $c:[\kappa]^{2} \rightarrow \theta$ as in the proof of Theorem 4.9 by setting, for all $\alpha<\beta<\kappa$,

$$
c(\alpha, \beta):=\max \{h(\xi) \mid \xi \in \operatorname{Im}(\operatorname{tr}(\alpha, \beta))\} .
$$

We claim that $c$ witnesses $\mathrm{U}(\kappa, \kappa, \theta, \chi)$ and prove this by verifying Clause (2) of Lemma 4.2. To this end, fix a family $\mathcal{A} \subseteq[\kappa]^{<\chi}$ consisting of $\kappa$-many pairwise disjoint sets, a club $D$ in $\kappa$, and a color $i<\theta$. We will find $\gamma \in D, a \in \mathcal{A}$, and $\epsilon<\gamma$ such that

- $\gamma<a$;

- for all $\alpha \in(\epsilon, \gamma)$ and all $\beta \in a$, we have $c(\alpha, \beta)>i$.

Since $\Gamma:=h^{-1}\{i+1\} \backslash(\tau+1)$ is in $\mathcal{I}^{+}$, we may fix $\delta \in S \backslash(\tau+1)$ such that $\sup \left(\operatorname{nacc}\left(e_{\delta}\right) \cap D \cap \Gamma\right)=\delta$. Fix an arbitrary $a \in \mathcal{A}$ with $\delta<a$, and set

- $T:=\left\{\xi \in \bigcup_{\beta \in a} \operatorname{Im}(\operatorname{tr}(\delta, \beta)) \mid \delta \in C_{\xi}\right\}$;

- $C:=C_{\delta} \cup \bigcup\left\{C_{\xi} \mid \xi \in T\right\}$;

- $\Lambda:=\sup \left\{\lambda_{2}^{k}(\delta, \beta), \sup \left(e_{\delta} \backslash C_{\xi}\right) \mid \beta \in a, \xi \in T\right\}$.

For all $\xi \in T$, since $C_{\xi} \cap S \neq \emptyset$, we infer that $\xi \notin \operatorname{Reg}(\kappa), \sup \left(e_{\delta} \backslash C_{\xi}\right)<\delta$, and $\left|C_{\xi}\right|<\min \left(C_{\xi}\right)<\delta$. In addition, $\left|C_{\delta}\right|=\sigma<\tau<\delta$ and $|a|<\chi \leq \sigma=\operatorname{cf}(\delta)$, so it 
follows that both $|C|$ and $\Lambda$ are less than $\delta$. Pick $\gamma \in \operatorname{nacc}\left(e_{\delta}\right) \cap D \cap \Gamma$ large enough so that $\gamma>\Lambda$ and $\operatorname{cf}(\gamma)>\max \{|C|, \chi\}$, and hence $\epsilon:=\max \{\Lambda, \sup (C \cap \gamma)\}$ is less than $\gamma$.

We claim that $\gamma, a$, and $\epsilon$ are as desired. To this end, let $\alpha \in(\epsilon, \gamma)$ and $\beta \in a$ be arbitrary. We have

$$
\lambda_{2}(\delta, \beta) \leq \Lambda \leq \epsilon<\alpha<\gamma<\delta<\beta,
$$

so, by Lemma 4.6, $\rho_{2}(\delta, \beta) \sqsubseteq \rho_{2}(\alpha, \beta)$. We claim that $\gamma \in \operatorname{Im}(\operatorname{tr}(\alpha, \beta))$. Set $\ell:=\rho_{2}(\delta, \beta)$, and consider the following two cases.

- If $\delta \in \operatorname{nacc}\left(C_{\operatorname{tr}(\delta, \beta)(\ell-1)}\right)$, then, since

$$
[\alpha, \delta) \cap C_{\operatorname{tr}(\delta, \beta)(\ell-1)} \subseteq\left(\lambda_{2}(\delta, \beta), \delta\right) \cap C_{\operatorname{tr}(\delta, \beta)(\ell-1)}=\emptyset,
$$

we have $\operatorname{tr}(\alpha, \beta)(\ell)=\min \left(C_{\operatorname{tr}(\alpha, \beta)(\ell-1)} \backslash \alpha\right)=\min \left(C_{\operatorname{tr}(\delta, \beta)(\ell-1)} \backslash \alpha\right)=\delta$. It follows that $C_{\operatorname{tr}(\alpha, \beta)(\ell)}=C_{\delta}$, so, since $\gamma \in e_{\delta} \backslash(\Lambda+1) \subseteq C_{\delta} \subseteq C$, we have

$$
[\alpha, \gamma) \cap C_{\operatorname{tr}(\alpha, \beta)(\ell)} \subseteq(\epsilon, \gamma) \cap C=\emptyset
$$

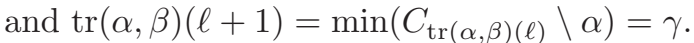

- If $\delta \in \operatorname{acc}\left(C_{\operatorname{tr}(\delta, \beta)(\ell-1)}\right)$, then $\gamma \in e_{\delta} \backslash(\Lambda+1) \subseteq C_{\operatorname{tr}(\delta, \beta)(\ell-1)} \subseteq C$, so $[\alpha, \gamma) \cap$ $C_{\operatorname{tr}(\delta, \beta)(\ell-1)}=\emptyset$ and $\operatorname{tr}(\alpha, \beta)(\ell)=\min \left(C_{\operatorname{tr}(\delta, \beta)(\ell-1)} \backslash \alpha\right)=\gamma$.

So, in either case, $\gamma \in \operatorname{Im}(\operatorname{tr}(\alpha, \beta))$, and hence $c(\alpha, \beta) \geq h(\gamma)>i$, as desired.

\section{Concluding Remarks}

(1) Theorem 1 of [Rin12] states that if $\lambda$ is a singular cardinal, $\theta \leq \lambda^{+}$, and $\operatorname{Pr}_{1}\left(\lambda^{+}, \lambda^{+}, \theta, \chi\right)$ holds for $\chi=2$, then $\operatorname{Pr}_{1}\left(\lambda^{+}, \lambda^{+}, \theta, \chi\right)$ holds also for $\chi=\operatorname{cf}(\lambda)$. Theorem 2.11 above implies that the latter is optimal and cannot be improved to $\chi=\operatorname{cf}(\lambda)^{+}$. Specifically, if $\lambda$ is a singular limit of strongly compact cardinals, then $\operatorname{Pr}_{1}\left(\lambda^{+}, \lambda^{+}, \lambda^{+}, 2\right)$ holds, ${ }^{1}$ but $\operatorname{Pr}_{1}\left(\lambda^{+}, \lambda^{+}, \lambda^{+}, \operatorname{cf}(\lambda)^{+}\right)$fails.

(2) In light of Lemma 2.4 and Theorem 4.16(3), we ask whether $\mathrm{U}\left(\lambda^{+}, 2, \theta, 2\right)$ for every pair of infinite cardinals $\theta \leq \lambda$, the instance $\mathrm{U}\left(\lambda^{+}, 2, \theta, 2\right)$ implies $\mathrm{U}\left(\lambda^{+}, \lambda^{+}, \theta, \operatorname{cf}(\lambda)\right)$.

(3) We do not know whether it is the case that, in ZFC, any true instance $\mathrm{U}(\kappa, \kappa, \ldots)$ may be witnessed by a closed coloring.

(4) We wonder whether Subsection 4.5 can be expanded to say more on instances of $\mathrm{U}(\kappa, \kappa, \ldots)$ in which $\kappa$ is an inaccessible cardinal of the form $\operatorname{cf}\left(2^{\nu}\right)$.

(5) In view of Fact 2.10, we conjecture that $\kappa$ is weakly compact iff $\mathrm{U}(\kappa, 2, \theta, 2)$ fails for all $\theta \in \operatorname{Reg}(\kappa)$. Recalling [Tod07, Question 8.1.4], we furthermore conjecture that $\kappa$ is weakly compact iff $\mathrm{U}(\kappa, 2, \omega, 2)$ fails.

\section{ACKNOWLEDGMENTS}

The results of this paper were presented by the first author at the Set Theory, Model Theory and Applications conference in Eilat, April 2018, and at the SETTOP 2018 conference in Novi Sad, July 2018, and by the second author at the $11^{\text {th }}$ Young Set Theory workshop in Lausanne, June 2018. We thank the organizers for the warm hospitality.

\footnotetext{
${ }^{1}$ By [EHR65], if $2^{\lambda}=\lambda^{+}$, then $\operatorname{Pr}_{1}\left(\lambda^{+}, \lambda^{+}, \lambda^{+}, 2\right)$ holds.
} 


\section{REFERENCES}

[BR19] Ari Meir Brodsky and Assaf Rinot. Distributive Aronszajn trees. Fund. Math., 2019. Accepted March 2018. Preprint available at http://www. assafrinot.com/paper/29.

[CL17] Sean Cox and Philipp Lücke. Characterizing large cardinals in terms of layered posets. Ann. Pure Appl. Logic, 168(5):1112-1131, 2017.

[EHR65] P. Erdős, A. Hajnal, and R. Rado. Partition relations for cardinal numbers. Acta Math. Acad. Sci. Hungar., 16:93-196, 1965.

[Eis10] Todd Eisworth. Club-guessing, stationary reflection, and coloring theorems. Ann. Pure Appl. Logic, 161(10):1216-1243, 2010.

[ES09] Todd Eisworth and Saharon Shelah. Successors of singular cardinals and coloring theorems II. Journal of Symbolic Logic, 74:1287-1309, 2009.

[FR17] David Fernandez-Breton and Assaf Rinot. Strong failures of higher analogs of Hindman's theorem. Trans. Amer. Math. Soc., 369(12):8939-8966, 2017.

[Gal80] Fred Galvin. Chain conditions and products. Fund. Math., 108(1):33-48, 1980.

[Hof13] Douglas J. Hoffman. A Coloring Theorem for Inaccessible Cardinals. ProQuest LLC, Ann Arbor, MI, 2013. Thesis (Ph.D.)-Ohio University.

[Ina17] Tanmay Inamdar. An example of a non-existent forcing axiom. 2017. Unpublished note.

[LHL19] Chris Lambie-Hanson and Philipp Lücke. Squares, ascent paths, and chain conditions. J. Symbolic Logic, 2019. Accepted August 2018. Preprint available at http://arxiv.org/abs/1709.04537.

[LS81] Richard Laver and Saharon Shelah. The $\aleph_{2}$-Souslin hypothesis. Trans. Amer. Math. Soc., 264(2):411-417, 1981.

[Rin10] Assaf Rinot. A relative of the approachability ideal, diamond and non-saturation. $J$. Symbolic Logic, 75(3):1035-1065, 2010.

[Rin11] Assaf Rinot. Jensen's diamond principle and its relatives. In Set Theory and Its Applications, volume 533 of Contemp. Math., pages 125-156. Amer. Math. Soc., Providence, RI, 2011.

[Rin12] Assaf Rinot. Transforming rectangles into squares, with applications to strong colorings. Adv. Math., 231(2):1085-1099, 2012.

[Rin14] Assaf Rinot. Chain conditions of products, and weakly compact cardinals. Bull. Symb. Log., 20(3):293-314, 2014.

[RS17] Assaf Rinot and Ralf Schindler. Square with built-in diamond-plus. J. Symbolic Logic, 82(3):809-833, 2017.

[She78] S. Shelah. A weak generalization of MA to higher cardinals. Israel J. Math., 30(4):297306, 1978.

[She88] Saharon Shelah. Successors of singulars, cofinalities of reduced products of cardinals and productivity of chain conditions. Israel J. Math., 62(2):213-256, 1988.

[She94] Saharon Shelah. There are Jonsson algebras in many inaccessible cardinals. In Cardinal Arithmetic, volume 29 of Oxford Logic Guides. Oxford University Press, 1994.

[She97] Saharon Shelah. Colouring and non-productivity of $\aleph_{2}$-cc. Annals of Pure and Applied Logic, 84:153-174, 1997.

[She10] Saharon Shelah. Diamonds. Proceedings of the American Mathematical Society, 138:2151-2161, 2010. 0711.3030.

[SS82] S. Shelah and L. Stanley. Generalized Martin's axiom and Souslin's hypothesis for higher cardinals. Israel J. Math., 43(3):225-236, 1982.

[Tal94] Franklin D. Tall. Some applications of a generalized Martin's axiom. Topology Appl., 57(2-3):215-248, 1994.

[Tod87] Stevo Todorcevic. Partitioning pairs of countable ordinals. Acta Math., 159(3-4):261294, 1987.

[Tod07] Stevo Todorcevic. Walks on Ordinals and Their Characteristics, volume 263 of Progress in Mathematics. Birkhäuser Verlag, Basel, 2007. 
Department of Mathematics, Bar-Ilan University, Ramat-Gan 5290002, Israel.

Current address: Department of Mathematics and Applied Mathematics, Virginia Commonwealth University, Richmond, VA 23284, USA

URL: http://people.vcu.edu/ ${ }^{c b l a m b i e h a n s o ~}$

Department of Mathematics, Bar-Ilan University, Ramat-Gan 5290002, Israel.

$U R L:$ http://www.assafrinot.com 